\title{
Peculiar orbits and asymmetries in extreme trans-Neptunian space
}

\author{
C. de la Fuente Marcos ${ }^{1 \star}$ and R. de la Fuente $\operatorname{Marcos}^{2}$ \\ ${ }^{1}$ Universidad Complutense de Madrid, Ciudad Universitaria, E-28040 Madrid, Spain \\ ${ }^{2}$ AEGORA Research Group, Facultad de Ciencias Matemáticas, Universidad Complutense de Madrid, Ciudad Universitaria, E-28040 Madrid, Spain
}

Accepted 2021 June 12. Received 2021 June 10; in original form 2021 January 5

\begin{abstract}
It is still an open question how the Solar system is structured beyond 100 au from the Sun. Our understanding of this vast region remains very limited and only recently we have become aware of the existence there of a group of enigmatic bodies known as the extreme transNeptunian objects (ETNOs) that have large orbits with perihelia beyond the orbit of Neptune. Four ETNOs — Sedna, Leleakuhonua, 2012 VP $_{113}$, and $2013 \mathrm{SY}_{99}$ - have perihelia beyond $50 \mathrm{au}$. The study of the ETNOs may provide much needed information on how this remote region is organized. Here, we apply machine-learning techniques to the sample of 40 known ETNOs to identify statistically significant clusters that may signal the presence of true dynamical groupings and study the distribution of the mutual nodal distances of the known ETNOs that measure how close two orbits can get to each other. Machine-learning techniques show that the known ETNOs may belong to four different populations. Results from the analysis of the distribution of nodal distances show that 41 per cent of the known ETNOs have at least one mutual nodal distance smaller than 1.45 au (1st percentile of the distribution), perhaps hinting at past interactions. In this context, the peculiar pair of ETNOs made of $505478\left(2013 \mathrm{UT}_{15}\right)$ and $2016 \mathrm{SG}_{58}$ has a mutual ascending nodal distance of 1.35 au at 339 au from the Sun. In addition, the known ETNOs exhibit a highly statistically significant asymmetry between the distributions of object pairs with small ascending and descending nodal distances that might be indicative of a response to external perturbations.
\end{abstract}

Key words: methods: statistical - celestial mechanics - minor planets, asteroids: general Kuiper belt: general - Oort Cloud.

\section{INTRODUCTION}

Our degree of understanding of the structure of the Solar system beyond 100 au from the Sun remains very limited. In order for this situation to change, we have to find and study objects with orbits not only much larger than those of typical members of the transNeptunian or Kuiper belt, but also with perihelia increasingly farther from Neptune so their trajectories are only weakly affected by the gravitational pull of the giant planets. The first object fitting into these rather general requirements, $148209\left(2000 \mathrm{CR}_{105}\right)$, was discovered in February 2000 at Lowell Observatory during a survey of the trans-Neptunian belt (Millis et al. 2000; Gladman et al. 2001), signalling the presence of an extended scattered structure well beyond Pluto.

A trickle of related discoveries has continued throughout the 21 st century, in spite of this being an intrinsically challenging task. Major milestones in this continuing effort have been the discoveries of 90377 Sedna $\left(2003 \mathrm{VB}_{12}\right.$ ) in 2003 (Brown, Trujillo \& Rabinowitz 2004) and of $2012 \mathrm{VP}_{113}$ in 2012 (Trujillo \& Sheppard 2014). Although the distinctive nature of this group of objects relative to that of the already well-understood scattered disc was soon

^ E-mail: nbplanet@ucm.es recognized (see e.g. Gladman et al. 2002; Kenyon \& Bromley 2004; Morbidelli \& Levison 2004; Stern 2005), the origin and evolution of this population (or populations) is far from well established.

Here, we adopt the naming convention featured in Trujillo \& Sheppard (2014): extreme (outer) Solar system bodies have perihelion distances, $q>30$ au and semimajor axes, $a>150$ au. These boundaries in $q$ and $a$ are dynamically motivated: $q>30$ au implies that the ETNOs cannot experience close encounters with Neptune and $a>150$ au makes a resonant engagement with Neptune unlikely (see e.g. Clement \& Sheppard 2021). Such a group of minor bodies was designated as exterior trans-Neptunian objects or ETNOs by Rickman et al. (2004) and extreme trans-Neptunian objects (also ETNOs) by de la Fuente Marcos \& de la Fuente Mar$\cos$ (2014). In the following, we will use the term 'extreme transNeptunian objects' (ETNOs) to refer to Trujillo and Sheppard's extreme outer Solar system bodies (those with $a>150$ au and $q>30 \mathrm{au})$.

The ETNOs are unlikely to be captured in distant $1: N$ meanmotion resonances with Neptune: the farthest ones with known objects in them are 1:9 at $a \sim 130$ au with $2007 \mathrm{TC}_{434}$ and $2015 \mathrm{KE}_{172}$ (Volk et al. 2018), and probably 1:10 at $a \sim 140$ au with 533563 $\left(2014 \mathrm{JW}_{80}\right)$ and $1: 11$ at $a \sim 150$ au with $543735\left(2014 \mathrm{OS}_{394}\right)$ as pointed out by Clement \& Sheppard (2021). Resonant objects in 


\section{C. de la Fuente Marcos and R. de la Fuente Marcos}

the 1:12 mean-motion resonance with Neptune at $a \sim 157$ au and beyond may not exist although Clement \& Sheppard (2021) showed reasonable numbers of captures as far out as the 1:14, see also the discussion in Gallardo (2006) regarding the 1:18, 1:19 and 1:20 mean-motion resonances with Neptune. ${ }^{1,2}$ The topic of Neptune's resonances in the scattered disc has already been extensively studied and it is relatively well understood within the context of the known Solar system (see e.g. Lykawka \& Mukai 2007; Kaib \& Sheppard 2016; Nesvorný, Vokrouhlický \& Roig 2016; Saillenfest et al. 2017a; Lan \& Malhotra 2019; Gallardo 2020). However, the issue of capture of small bodies in distant mean-motion resonances with Neptune remains an open question. Volk, Malhotra, \& Graham (2021) have recently explored the $1: N, 2: N$ and $3: N$ mean-motion resonances with Neptune out to $a=550$ au at a wide range of perihelion distances $(q=33-60 \mathrm{au})$ within the context of the restricted three-body problem to find out that at large $a$, the surviving libration zones of Neptune's strongest resonances are generally wider for larger $q$ due to less crowding from weaker neighbouring resonances, although the sticking times tend to be shorter as one moves further out (see e.g. Gallardo 2006).

The ETNOs discovered so far (see Table 1) move in rather elongated orbits and have perihelion distances beyond $30 \mathrm{au}$; these unusual properties make them particularly hard to find. In fact, the ETNOs have only been found at perihelion or very near it, leading to a very distinctive pattern regarding how orbital properties relate to discovery locations: their equatorial coordinates at discovery time strongly constraint the orientations of their orbits in space. Orbits are defined by the values of semimajor axis (that controls orbital size), eccentricity, $e$ (that controls shape), and those of the angular elements - inclination, $i$, longitude of the ascending node, $\Omega$, and argument of perihelion, $\omega-$ that control the orientation of the orbit in space.

Figure 1 in de la Fuente Marcos \& de la Fuente Marcos (2014) shows that the sizes and shapes of the orbits of the ETNOs (panels $\mathrm{B}$ and $\mathrm{C}$ in the figure) do not depend on the position of the object at discovery, but $i, \Omega$ and $\omega$ (panels $\mathrm{D}, \mathrm{E}$ and $\mathrm{F}$ ) do. This means that the way observations have been conducted (i.e. where the discoveries are being made) affects the observed distributions of the angular elements (the true distributions are, at this point, unknown). This observational bias implies that ETNOs discovered within a few degrees of each other must have similar orbital orientations. This unfortunate circumstance has led to great controversy regarding the presence (of any kind) of clustering in $\Omega, \omega$, and longitude of perihelion, $\varpi=\Omega+\omega$ (see e.g. Batygin \& Brown 2016; de la Fuente Marcos \& de la Fuente Marcos 2016; Brown 2017; Shankman et al. 2017; Batygin et al. 2019; Sheppard et al. 2019; Bernardinelli et al. 2020a; Batygin \& Brown 2021; Napier et al. 2021).

However, the known ETNOs have been discovered by several, unrelated surveys and the samples produced by different surveys with different depths and different pointing histories would result in sets of observables with different sampled distributions, particularly in the case of $i, \Omega$ and $\omega$. In addition, the overall distributions of the entire observed sample in the case of $a$ and $e$ are expected to resemble in some way the true distributions. On the other hand, it is often unwarrantedly assumed that the known ETNOs represent a single, monolithic population when the reality could be more complex, as it is in the better-studied trans-Neptunian belt with classical, resonant, scattered and detached objects (see e.g. Adams et al. 2014; Bannister et al. 2018; Khain et al. 2020). Whether or not there is a plausible single population of ETNOs remains an open question and here we explore possible answers by applying machine-learning techniques to the sample of 40 known ETNOs to study how are they arranged in orbital parameter space. This paper is organized as follows. In Section 2, we discuss data and methods. Machine-learning techniques, in the form of the $k$-means++ algorithm implemented in the Python library Scikit-learn, are applied in Section 3 to three-dimensional datasets to evaluate the presence of any significant clustering in the sample. Our findings are tested against the distribution of their mutual nodal distances that measures how close two orbits can get to each other in Section 4. Poles and perihelia that define the orientation of the orbits in space are studied in Section 5. Our results are discussed in Section 6 and our conclusions are summarized in Section 7.

\section{DATA AND METHODS}

Here, we work with publicly available data from Jet Propulsion Laboratory's (JPL) Small-Body Database (SBDB) ${ }^{3}$ and HORIZONS on-line solar system data and ephemeris computation service, ${ }^{4}$ both provided by the Solar System Dynamics Group (Giorgini 2011, 2015). The HORIZONS ephemeris system has recently been updated to replace the DE430/431 planetary ephemeris, used since 2013, with the new DE440/441 solution (Park et al. 2021) and sixteen most massive small-body perturbers. The new DE440/441 general-purpose planetary solution includes seven additional years of ground and space-based astrometric data, data calibrations, and dynamical model improvements, most significantly involving Jupiter, Saturn, Pluto, and the Kuiper Belt (Park et al. 2021). DE440 covers the years $1550-2650$ while DE441 is tuned to cover a time range of $-13,200$ to $+17,191$ years (Park et al. 2021). The most visible change with this update may be in the ephemerides expressed with respect to the Solar system barycentre. There is a time-varying shift of $\sim 100 \mathrm{~km}$ in DE441's barycentre relative to DE431 due to the inclusion of 30 new Kuiper-belt masses, and the Kuiper Belt ring mass (Park et al. 2021).

In the following, we use barycentric elements because, within the context of the ETNOs, barycentric orbit determinations account better for their changing nature as Jupiter follows its $12 \mathrm{yr}$ orbit around the Sun. Although the values of semimajor axis and eccentricity in barycentric orbit determinations may differ from those of heliocentric ones (for the same object), the ones of the angular elements are nearly the same in both cases (de la Fuente Marcos \& de la Fuente Marcos 2016). Table 1 shows the input data used in our analyses that include orbital elements and $1 \sigma$ uncertainties (if known) of 40 known ETNOs. The data (as of 10-June-2021) are referred to epoch 2459200.5 Barycentric Dynamical Time (TDB) and they have been retrieved from JPL's SBDB and HORIZONS using tools provided by the Python package Astroquery (Ginsburg et al. 2019). Only four known ETNOs have perihelia, $q=a(1-e)$, beyond 50 au: 90377 Sedna $\left(2003 \mathrm{VB}_{12}\right), 541132$ Leleakuhonua (2015 $\mathrm{TG}_{387}$ ), $2012 \mathrm{VP}_{113}$, and $2013 \mathrm{SY}_{99}$. The current orbit determination of $2020 \mathrm{KV}_{11}$ does not include $1 \sigma$ uncertainties. The orbit determination of $2003 \mathrm{SS}_{422}$ does include uncertainties, but it is the least precise in the sample shown in Table 1.

In order to analyse the results, we produce histograms using $\begin{array}{ll}1 & \text { http://www.fisica.edu.uy/ gallardo/atlas/2020/atlas50to225i10col.png } \\ 2 & \text { http://www.fisica.edu.uy/gallardo/atlas/2020/atlas0to230.png }\end{array}$
3 https://ssd.jpl.nasa.gov/sbdb.cgi

4 https://ssd.jpl.nasa.gov/?horizons 
Table 1. Barycentric orbital elements, $1 \sigma$ uncertainties (if known), results of the clustering analysis presented in Section 3 (cluster number), and number of mutual nodal distances $(X)$ below the 1st percentil of $\Delta_{+}, 1.450 \mathrm{au}$. The orbit determinations have been computed at epoch JD 2459200.5 that corresponds to 00:00:00.000 TDB on 2020 Dec 17 (J2000.0 ecliptic and equinox). The current orbit determination of $2020 \mathrm{KV}_{11}$ does not include uncertainties. Input data source: JPL's SBDB.

\begin{tabular}{|c|c|c|c|c|c|c|c|}
\hline Object & $a_{\mathrm{b}}(\mathrm{au})$ & $e_{\mathrm{b}}$ & $i_{\mathrm{b}}\left({ }^{\circ}\right)$ & $\Omega_{\mathrm{b}}\left({ }^{\circ}\right)$ & $\omega_{\mathrm{b}}\left({ }^{\circ}\right)$ & cluster & $x$ \\
\hline $82158\left(2001 \mathrm{FP}_{185}\right)$ & $215.55 \pm 0.04$ & $0.84110 \pm 0.00003$ & $30.80030 \pm 0.00003$ & $179.35849 \pm 0.00004$ & $6.8745 \pm 0.0005$ & 0 & 0 \\
\hline $148209\left(2000 \mathrm{CR}_{105}\right)$ & $221.9 \pm 0.6$ & $0.8012 \pm 0.0006$ & $22.7558 \pm 0.0006$ & $128.2858 \pm 0.0003$ & $316.691 \pm 0.012$ & 0 & 3 \\
\hline 474640 Alicanto $\left(2004 \mathrm{VN}_{112}\right)$ & $328 \pm 2$ & $0.8556 \pm 0.0007$ & $25.5480 \pm 0.0003$ & $66.0222 \pm 0.0004$ & $326.988 \pm 0.009$ & 0 & 3 \\
\hline $496315\left(2013 \mathrm{GP}_{136}\right)$ & $150.2 \pm 0.2$ & $0.7269 \pm 0.0004$ & $33.5389 \pm 0.0006$ & $210.72725 \pm 0.00011$ & $42.58 \pm 0.04$ & 0 & 1 \\
\hline $508338\left(2015 \mathrm{SO}_{20}\right)$ & $164.78 \pm 0.02$ & $0.79871 \pm 0.00002$ & $23.41068 \pm 0.00003$ & $33.63414 \pm 0.00002$ & $354.8271 \pm 0.0005$ & 0 & 0 \\
\hline $2013 \mathrm{RF}_{98}$ & $365 \pm 14$ & $0.901 \pm 0.004$ & $29.538 \pm 0.003$ & $67.636 \pm 0.005$ & $311.7 \pm 0.7$ & 0 & 1 \\
\hline $2013 \mathrm{UH}_{15}$ & $174 \pm 9$ & $0.799 \pm 0.011$ & $26.080 \pm 0.006$ & $176.542 \pm 0.007$ & $282.9 \pm 0.3$ & 0 & 0 \\
\hline $2014 \mathrm{WB}_{556}$ & $280 \pm 3$ & $0.8475 \pm 0.0013$ & $24.1575 \pm 0.0002$ & $114.891 \pm 0.003$ & $235.33 \pm 0.06$ & 0 & 1 \\
\hline $2015 \mathrm{BP}_{519}$ & $449 \pm 9$ & $0.921 \pm 0.002$ & $54.11068 \pm 0.00010$ & $135.213 \pm 0.002$ & $348.06 \pm 0.03$ & 0 & 0 \\
\hline $2015 \mathrm{KH}_{163}$ & $153.0 \pm 0.6$ & $0.7390 \pm 0.0011$ & $27.1377 \pm 0.0014$ & $67.5728 \pm 0.0006$ & $230.82 \pm 0.05$ & 0 & 1 \\
\hline $2016 \mathrm{TP}_{120}$ & $175 \pm 25$ & $0.77 \pm 0.04$ & $32.6395 \pm 0.0006$ & $126.73 \pm 0.03$ & $351.0 \pm 0.4$ & 0 & 0 \\
\hline $523622\left(2007 \mathrm{TG}_{422}\right)$ & $502.5 \pm 0.3$ & $0.92923 \pm 0.00003$ & $18.59537 \pm 0.00003$ & $112.91052 \pm 0.00012$ & $285.6641 \pm 0.0009$ & 1 & 1 \\
\hline 541132 Leleakuhonua (2015 $\left.\mathrm{TG}_{387}\right)$ & $1090 \pm 126$ & $0.940 \pm 0.006$ & $11.6714 \pm 0.0006$ & $300.995 \pm 0.007$ & $118.0 \pm 0.3$ & 1 & 0 \\
\hline $2010 \mathrm{~GB}_{174}$ & $349 \pm 7$ & $0.861 \pm 0.003$ & $21.563 \pm 0.002$ & $130.716 \pm 0.008$ & $347.27 \pm 0.08$ & 1 & 0 \\
\hline $2013 \mathrm{FT}_{28}$ & $292 \pm 2$ & $0.8509 \pm 0.0008$ & $17.3754 \pm 0.0014$ & $217.722 \pm 0.002$ & $40.65 \pm 0.05$ & 1 & 1 \\
\hline $2013 \mathrm{RA}_{109}$ & $463 \pm 2$ & $0.9006 \pm 0.0004$ & $12.39970 \pm 0.00008$ & $104.800 \pm 0.004$ & $262.92 \pm 0.02$ & 1 & 0 \\
\hline 2013 SY $_{99}$ & $736 \pm 28$ & $0.932 \pm 0.002$ & $4.2253 \pm 0.0012$ & $29.510 \pm 0.005$ & $32.18 \pm 0.11$ & 1 & 0 \\
\hline $2013 \mathrm{SL}_{102}$ & $314.4 \pm 0.7$ & $0.8787 \pm 0.0003$ & $6.50492 \pm 0.00008$ & $94.731 \pm 0.006$ & $265.50 \pm 0.06$ & 1 & 2 \\
\hline $2014 \mathrm{FE}_{72}$ & $1621 \pm 445$ & $0.978 \pm 0.009$ & $20.633 \pm 0.003$ & $336.830 \pm 0.005$ & $133.95 \pm 0.06$ & 1 & 0 \\
\hline $2014 \mathrm{SR}_{349}$ & $299 \pm 21$ & $0.841 \pm 0.011$ & $17.968 \pm 0.002$ & $34.884 \pm 0.015$ & $341.3 \pm 0.6$ & 1 & 0 \\
\hline $2015 \mathrm{GT}_{50}$ & $311 \pm 3$ & $0.8766 \pm 0.0011$ & $8.7950 \pm 0.0012$ & $46.064 \pm 0.003$ & $128.99 \pm 0.11$ & 1 & 0 \\
\hline $2015 \mathrm{KG}_{163}$ & $680 \pm 5$ & $0.9404 \pm 0.0004$ & $13.9942 \pm 0.0012$ & $219.103 \pm 0.002$ & $32.10 \pm 0.10$ & 1 & 1 \\
\hline $2015 \mathrm{RX}_{245}$ & $423 \pm 5$ & $0.8924 \pm 0.0013$ & $12.138 \pm 0.002$ & $8.6052 \pm 0.0002$ & $65.12 \pm 0.05$ & 1 & 1 \\
\hline $445473\left(2010 \mathrm{VZ}_{98}\right)$ & $153.434 \pm 0.011$ & $0.77612 \pm 0.00002$ & $4.510577 \pm 0.000009$ & $117.3943 \pm 0.0003$ & $313.7285 \pm 0.0007$ & 2 & 0 \\
\hline $505478\left(2013 \mathrm{UT}_{15}\right)$ & $200.2 \pm 0.8$ & $0.7806 \pm 0.0010$ & $10.6521 \pm 0.0010$ & $191.9542 \pm 0.0004$ & $252.13 \pm 0.03$ & 2 & 3 \\
\hline $506479\left(2003 \mathrm{HB}_{57}\right)$ & $159.6 \pm 0.4$ & $0.7613 \pm 0.0005$ & $15.5004 \pm 0.0003$ & $197.8710 \pm 0.0004$ & $10.837 \pm 0.009$ & 2 & 0 \\
\hline $527603\left(2007 \mathrm{VJ}_{305}\right)$ & $192.00 \pm 0.05$ & $0.81675 \pm 0.00004$ & $11.98365 \pm 0.00007$ & $24.38250 \pm 0.00003$ & $338.3561 \pm 0.0009$ & 2 & 1 \\
\hline $2002 \mathrm{~GB}_{32}$ & $206.7 \pm 0.5$ & $0.8290 \pm 0.0004$ & $14.1921 \pm 0.0002$ & $177.0434 \pm 0.0003$ & $37.048 \pm 0.005$ & 2 & 1 \\
\hline $2003 \mathrm{SS}_{422}$ & $201 \pm 138$ & $0.8 \pm 0.2$ & $16.78 \pm 0.15$ & $151.0 \pm 0.2$ & $211 \pm 43$ & 2 & 0 \\
\hline $2005 \mathrm{RH}_{52}$ & $153.6 \pm 0.2$ & $0.7461 \pm 0.0003$ & $20.4456 \pm 0.0004$ & $306.1097 \pm 0.0009$ & $32.513 \pm 0.008$ & 2 & 0 \\
\hline $2015 \mathrm{RY}_{245}$ & $225 \pm 5$ & $0.861 \pm 0.003$ & $6.0306 \pm 0.0010$ & $341.532 \pm 0.006$ & $354.5 \pm 0.2$ & 2 & 0 \\
\hline $2016 \mathrm{GA}_{277}$ & $155 \pm 7$ & $0.768 \pm 0.011$ & $19.4211 \pm 0.0003$ & $112.84 \pm 0.02$ & $178.52 \pm 0.11$ & 2 & 0 \\
\hline $2016 \mathrm{QU}_{89}$ & $171.5 \pm 0.3$ & $0.7944 \pm 0.0004$ & $16.9757 \pm 0.0005$ & $102.898 \pm 0.004$ & $303.35 \pm 0.08$ & 2 & 1 \\
\hline $2016 \mathrm{QV}_{89}$ & $171.66 \pm 0.08$ & $0.76725 \pm 0.00012$ & $21.3874 \pm 0.0002$ & $173.2148 \pm 0.0007$ & $281.09 \pm 0.02$ & 2 & 0 \\
\hline $2016 \mathrm{SG}_{58}$ & $232.9 \pm 0.4$ & $0.8493 \pm 0.0003$ & $13.22090 \pm 0.00005$ & $118.978 \pm 0.002$ & $296.29 \pm 0.04$ & 2 & 3 \\
\hline $2018 \mathrm{AD}_{39}$ & $166 \pm 7$ & $0.767 \pm 0.012$ & $19.770 \pm 0.007$ & $330.091 \pm 0.014$ & $49.22 \pm 0.14$ & 2 & 0 \\
\hline $2018 \mathrm{VM}_{35}$ & $261 \pm 64$ & $0.83 \pm 0.05$ & $8.480 \pm 0.003$ & $192.41 \pm 0.06$ & $303 \pm 3$ & 2 & 0 \\
\hline $2020 \mathrm{KV}_{11}$ & 151.11022838 & 0.77980725 & 4.58080324 & 135.47431392 & 52.68155301 & 2 & - \\
\hline 90377 Sedna $\left(2003 V_{12}\right)$ & $506.4 \pm 0.2$ & $0.84954 \pm 0.00005$ & $11.928523 \pm 0.000004$ & $144.4012 \pm 0.0005$ & $311.286 \pm 0.003$ & 3 & 0 \\
\hline $2012 \mathrm{VP}_{113}$ & $261.9 \pm 1.5$ & $0.693 \pm 0.002$ & $24.053 \pm 0.002$ & $90.801 \pm 0.006$ & $293.9 \pm 0.4$ & 3 & 0 \\
\hline
\end{tabular}

the Matplotlib library (Hunter 2007) with sets of bins computed using NumPy (van der Walt, Colbert \& Varoquaux 2011; Harris et al. 2020) by applying the Freedman and Diaconis rule (Freedman \& Diaconis 1981). The clustering analyses presented here were obtained by applying machine-learning techniques provided by the Python library Scikit-learn (Pedregosa et al. 2011; Buitinck et al. 2013); kernel density estimations have been carried out using the Python library SciPy (Virtanen et al. 2020).

\section{CLUSTERING: RESULTS FROM THE $K$-MEANS++ ALGORITHM}

In this section, we have applied the unsupervised machine-learning algorithm $k$-means++ to the data discussed above to evaluate data clustering in barycentric $\left(a_{\mathrm{b}}, e_{\mathrm{b}}, i_{\mathrm{b}}\right)$ space (the other two angular elements are not used due to the bias concerns pointed out above). The $k$-means++ algorithm (Arthur \& Vassilvitskii 2007) performs a centroid-based analysis and it is an improved version of the classical $k$-means algorithm (see e.g. Steinhaus 1957; MacQueen 1967; Lloyd 1982). The implementation of $k$-means++ used here is the one included in the method fit that is part of the KMeans class provided by the Python library Scikit-learn (Pedregosa et al. 2011). The method fit_predict computes cluster centres and returns 
cluster labels or indexes. As the threshold to define the final clusters in the dataset, we have used the elbow method to determine the optimal value of clusters, $k$; we invoke the method fit with $k$ in the interval $(1,10)$ and select the value of $k$ that minimizes the sum of the distances of all data points to their respective cluster centres. As part of the data preparation process, we have scaled the dataset using standardisation or Z-score normalisation: found the mean and standard deviation for $a_{\mathrm{b}}, e_{\mathrm{b}}$ and $i_{\mathrm{b}}$, subtracted the relevant mean from each value, and then divided by its corresponding standard deviation. This was carried out by applying the method fit_transform that is part of the StandardScaler class provided by the Python library Scikit-learn (Pedregosa et al. 2011). Distance assignment between the objects in our sample assumes a Euclidean metric.

\subsection{Trans-Neptunian objects}

In order to evaluate the reliability of the results of the unsupervised machine-learning algorithm $k$-means ++ within the context of the trans-Neptunian populations, we have applied the sequence of steps described above to the (heliocentric orbital elements) dataset made of 3119 trans-Neptunian objects or TNOs with $q>30$ au. Our approach is essentially different from the one discussed by Smullen \& Volk (2020). Known TNOs are classified as classical, resonant, scattered and detached objects (see e.g. Adams et al. 2014; Bannister et al. 2018; Khain et al. 2020). Classical TNOs have $a \in(42,46)$ au with a cold component with $e \leqslant 0.1$ and $i \leqslant 5 \mathrm{deg}$ and a hot one with higher values of $e$ and $i$. Resonant TNOs are trapped in mean-motion resonances with Neptune. Scattered TNOs have $a \in(30,1000)$ au and $q \in(30,38)$ au. Detached TNOs have $a \geqslant 50$ au and $q \geqslant 40$ au. Figure 1 shows the results of the application of $k$-means++ and the elbow method to the TNO sample; results are different depending on the orbital parameters used to perform the multiparametric analysis although the three analyses produce four clusters. While unsupervised machinelearning techniques applied to orbital elements are not expected to reproduce precisely the results of classification algorithms based on extensive numerical simulations, they indeed show that four distinct populations may be present in the full TNO sample. Our results are purely statistical, without any input from $N$-body simulations. But, which (tri-parametric) dataset does produce the most consistent results when considering the actual dynamical classes pointed out above?

The left-hand side panels of Fig. 1 are the result of focusing on $(a, e, i)$ and fail to show the resonant TNOs as a separate dynamical class. The cold (aqua) and hot (azure) components of the classical TNOs are clearly identified albeit also including the resonant TNOs and perhaps others. Scattered and detached TNOs appear mixed (plum). The nine objects with the largest values of $a$ appear as a separate group (fuchsia). The central panels of Fig. 1 are for $(q, e, i)$. In this case, resonant TNOs (azure) are relatively well identified at $i<20 \mathrm{deg}$ and also the cold (fuchsia) and hot (aqua) components of the classical TNOs. Scattered and detached TNOs mingle (plum) as they did when we considered $(a, e, i)$. In our third analysis, the right-hand side panels of Fig. 1 show the results of focusing on $(a, q, i)$. The cold (aqua) component of the classical TNOs is clearly identified but the hot one appears mixed with scattered and detached TNOs (fuchsia). The resonant TNOs (azure) also appear mixed with scattered and detached TNOs. The nine objects with the largest values of $a$ are identified as a separate group (plum).

The virtual absence of TNOs moving in low-eccentricity, low- inclination orbits beyond about 50 au and visible in Fig. 1 was first noticed by Dones (1997), Gladman et al. (1998) and Jewitt, Luu \& Trujillo (1998). Chiang \& Brown (1999) coined the term 'Kuiper Cliff' to refer to such a feature and it has been used ever since to describe the unexpected drop-off in the fraction of TNOs following dynamically cold paths. The reality of the Kuiper Cliff has been demonstrated by multiple studies (see e.g. Chiang \& Jordan 2002; Buie et al. 2003; Chiang et al. 2003a; Bernstein et al. 2004; Larsen et al. 2007).

Although the results of the clustering analysis carried out with the $k$-means++ algorithm cannot reproduce the widely recognized dynamical classes inhabiting trans-Neptunian orbital parameter space, the analysis based on $(q, e, i)$ is perhaps the one providing the most approximate results. All the analyses fail to separate scattered and detached TNOs properly, which is to be expected as they have disperse distributions, not well suited to be resolved by any type of clustering algorithm.

\subsection{Extreme trans-Neptunian objects}

When applying the $k$-means++ algorithm and the elbow method to the ETNO dataset, we obtained four clusters that are shown in Fig. 2; a consistent result is obtained when heliocentric orbit determinations are used instead of barycentric ones. The central panels of Fig. 2 are for $(q, e, i)$ that produced the best matching for the full TNO sample in terms of dynamical groupings. In the following, we will focus on the results of this particular clustering analysis. The colours in Fig. 2 are linked to the number labels (cluster indexes) in Table 1: 0 in aqua, 1 in azur, 2 in plum, and 3 in fuchsia. The reference object for cluster 0 is $148209\left(2000 \mathrm{CR}_{105}\right)$, for cluster 1 is 541132 Leleakuhonua $\left(2015 \mathrm{TG}_{387}\right)$, the one for cluster 2 is $445473\left(2010 \mathrm{VZ}_{98}\right)$, and the reference object for cluster 3 is 90377 Sedna $\left(2003 \mathrm{VB}_{12}\right.$ ). There is an object, $2015 \mathrm{BP}_{519}$ (in cluster 0 , see Fig. 2, top panel), that is a statistical outlier in terms of the inclination value, $54^{\circ} 11068 \pm 0.00010$ (Becker et al. 2018; de la Fuente Marcos \& de la Fuente Marcos 2018a).

In our analysis, the Sednoids (those objects following Sednalike orbits) emerge as a statistically significant cluster (\#3 in fuchsia, Fig. 2, central panels), the smallest of the four found here, with just one member other than Sedna, $2012 \mathrm{VP}_{113}$. Although clusters 0 and 2 may be part of the scattered and detached TNO dynamical classes, cluster 1 may represent a separate population, perhaps linked to the so-called inner Oort Cloud (Hills 1981; Levison, Dones \& Duncan 2001). Both Leleakuhonua and 2013 SY $_{99}$ are members of cluster 1. Sedna, Leleakuhonua, $2012 \mathrm{VP}_{113}$, and 2013 SY $_{99}$ (barely) are the only known objects with $q>50$ au, i.e. beyond the Kuiper Cliff. The four statistically significant clusters may represent true dynamical groupings, but additional analyses are required before reaching a final conclusion regarding this issue. In any case, statistics alone strongly suggests that the known ETNOs do not belong to a single dynamical group but to a mixture of several, probably four, populations.

\section{NODAL DISTANCES}

If real, the four statistically significant clusters singled out in the previous section must produce consistent structures in other distributions of relevant orbital parameters. Here, we focus on the distribution of mutual nodal distances (absolute values) computed as described in Appendix A using data from Table 1. A small mutual nodal distance implies that in absence of protective mechanisms 

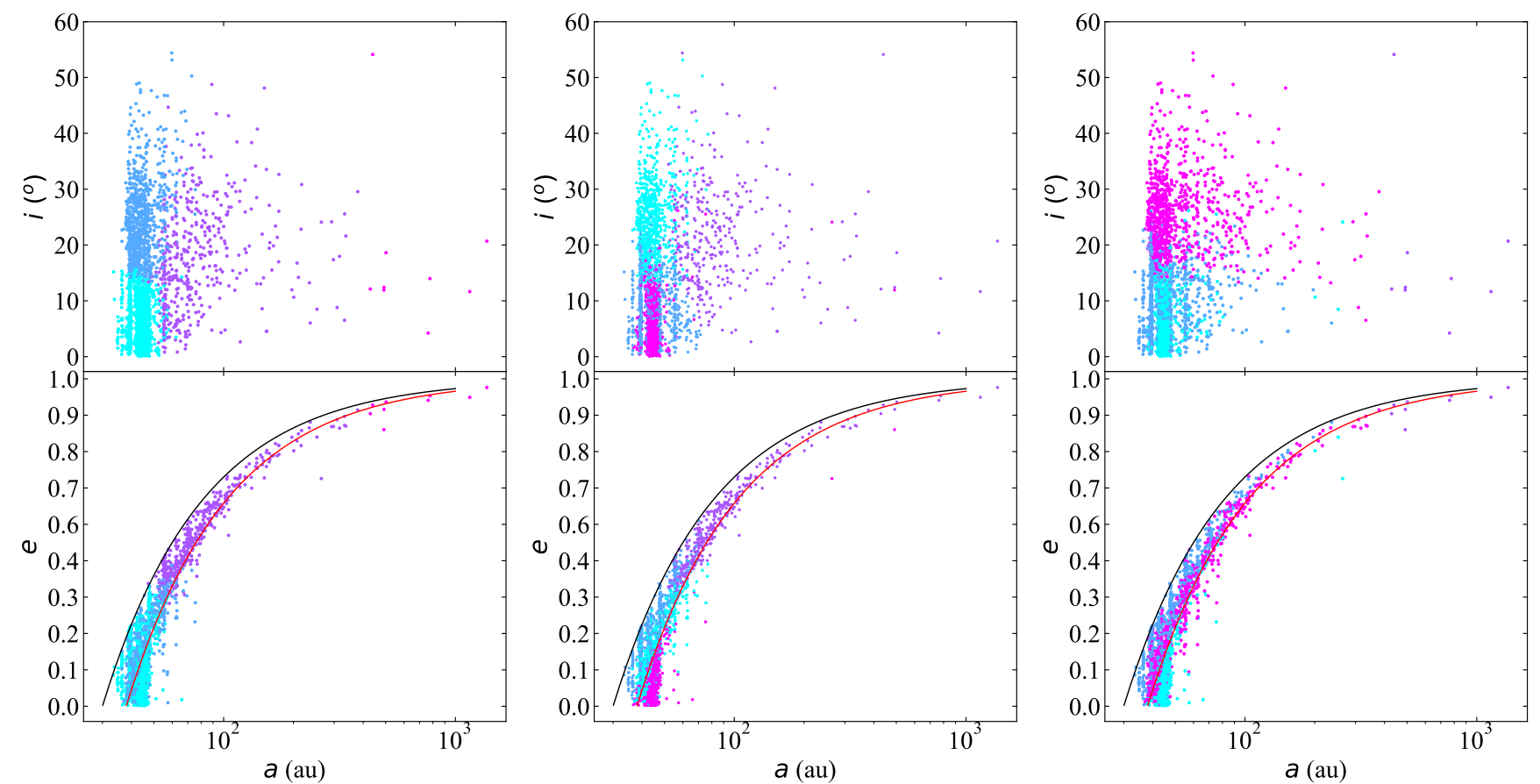

Figure 1. Colour-coded clusters generated by the $k$-means++ algorithm applied to the dataset made of 3119 trans-Neptunian objects (TNOs). The left-hand side panels correspond to the algorithm being applied to the dataset $(a, e, i)$; the central panels are for $(q, e, i)$; the right-hand side panels are for $(a, q, i)$. The black curve shows $q=30$ au and the red one $q=38$ au. In the left-hand side panels, the cold (aqua) and hot (azure) components of the classical TNOs are well separated by the algorithm, scattered and detached TNOs appear mixed (plum), and the nine objects with the largest values of $a$ appear as a separate group (fuchsia). In the central panels, the cold (fuchsia) and hot (aqua) components of the classical TNOs and the resonant TNOs (azure) are relatively well identified, scattered and detached TNOs appear mixed (plum). The right-hand side panels show the cold (aqua) component of the classical TNOs, the resonant TNOs (azure) appear mixed with scattered and detached TNOs that also mingle with the hot (fuchsia) component, and the nine objects with the largest values of $a$ appear as a separate group (plum).

such as mean-motion or secular resonances, the objects may experience close flybys and perhaps collisions. Discarding $2020 \mathrm{KV}_{11}$ because its orbit determination is still too uncertain, we have a sample of 39 ETNOs that produced 741 pairs (i.e. 1482 in total) of mutual nodal distances, $\Delta_{ \pm}$(the results for each pair come from a set of $10^{4}$ pairs of virtual ETNOs as described in Appendix A). The distribution of mutual nodal distances for the ascending nodes is shown in Fig. 3 and the one corresponding to the descending nodes is displayed in Fig. 4. These distributions have been computed using the data in Table 1 and considering the uncertainties in the orbit determinations as described in Appendix A.

Applying the $k$-means ++ algorithm to the $\Delta_{ \pm}$dataset as described in the previous section, we obtained Fig. 5 that again displays four clusters. The coincidence in the number of clusters is significant as the nodal distance analysis uses the uncertainties in the orbit determinations and includes all the orbital elements, not just $\left(a_{\mathrm{b}}, e_{\mathrm{b}}, i_{\mathrm{b}}\right)$. Finding four clusters in terms of nodes means that one of the clusters identified in the previous section is not interacting with the other three. The region in aqua in Fig. 5 corresponds to smaller values of $\Delta_{ \pm}$linked to orbits that in absence of protective resonant mechanisms may lead to relatively close flybys, the others include pairs of objects whose orbits cannot intersect as they are fully detached. We interpret this result as supportive of the presence of four true dynamical groups in the sample of 39 ETNOs. The Gaussian kernel density estimation in Fig. 6 shows that there are a number of pairs with unusually low values of the mutual nodal distances considering the large sizes of the orbits involved.
This is more clearly seen when considering Figs 7 and 8, where the nodal distances of each pair appear colour coded. In these figures, the ETNOs have been sorted ( $x$-axis from left to right, $y$ axis from bottom to top) as they appear in Table 1 and the statistically significant clusters identified in Section 3.2 have been indicated using dashed horizontal and vertical lines of colours consistent with those in Fig. 2, central panels. Figures 7 and 8 show an obvious asymmetry as there are far more low values of mutual ascending nodal distances (Fig. 7) than of the descending ones (Fig. 8). The figures show the median values of the mutual nodal distances that have been computed as described in Appendix A, taking into account the uncertainties in Table 1 by using a Monte Carlo approach. Figures 7 and 8 show that there are two types of ETNOs, those that follow orbits that pass rather close to the paths of other ETNOs and those in trajectories well detached from the rest. ETNOs in cluster 3, the Sednoids, do not pass close to any other known ETNOs. There are no known pairs with low values of both mutual nodal distances. Among the pairs with low values of one mutual nodal distance, only a few are made of ETNOs within the same cluster. Most low values appear in pairs that include members of clusters 0 and 2. Cluster 1 has comparatively less members in pairs with low values of the mutual nodal distances than clusters 0 and 2.

In order to identify severe outliers, we use the 1st percentile of the distribution (see e.g. Wall \& Jenkins 2012) that is $1.450 \pm 0.010$ au for $\Delta_{+}$and $2.335 \pm 0.014$ au for $\Delta_{-}$(the dispersions come from ten sets of Monte Carlo experiments). Only three 

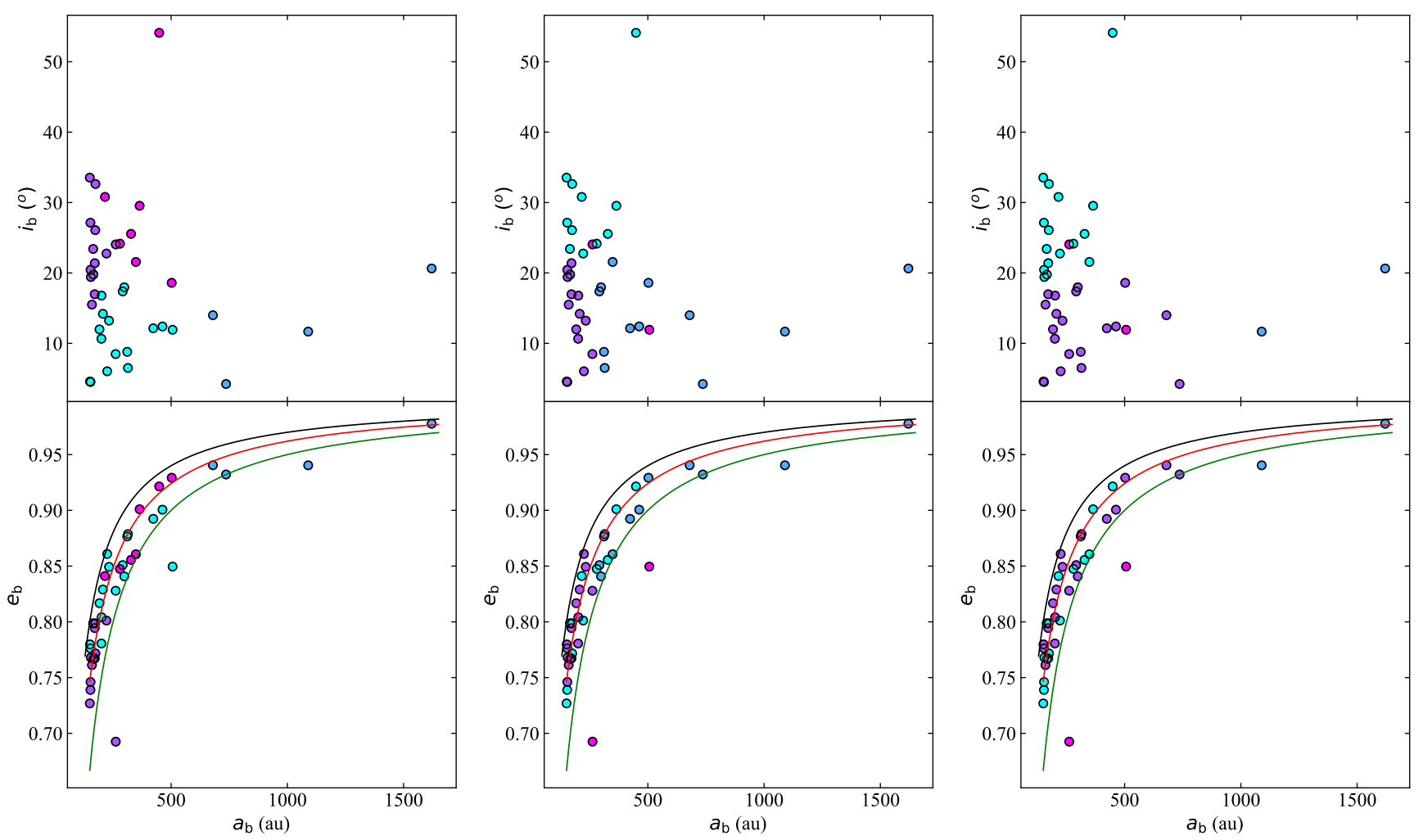

Figure 2. Colour-coded clusters generated by the $k$-means++ algorithm applied to the dataset made of 40 extreme trans-Neptunian objects (ETNOs). The left-hand side panels correspond to the algorithm being applied to the dataset $(a, e, i)$; the central panels are for $(q, e, i)$; the right-hand side panels are for $(a$, $q, i$ ), see the text for details. The black curve shows $q=30 \mathrm{au}$, the red one $q=38 \mathrm{au}$, and the green one $q=50$ au. Focusing on the clusters from the ( $q, e, i$ ) analysis: the aqua-coloured points (11) correspond to objects labelled as ' 0 ' in Table 1, the azur points (12) correspond to those labelled as ' 1 ', the plum points (15) correspond to those labelled as ' 2 ', and the fuchsia points (2) correspond to ETNOs labelled as ' 3 ' in the table.

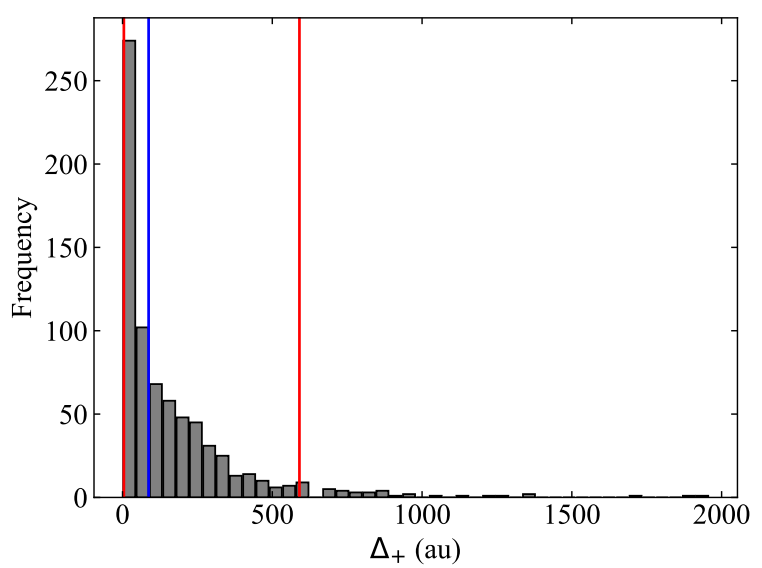

Figure 3. Distribution of mutual nodal distances for the ascending nodes of the sample of 39 ETNOs. The median is shown in blue and the 5th and 95th percentiles in red.

mutual nodal distance values out of the 1482 computed are below 0.5 au (probability of 0.0020 ) and 12 are below 1.450 au (probability of 0.0081 ), the 1 st percentile for $\Delta_{+}$. Out of these 12 pairs, eight have close ascending nodes and four have close descending nodes

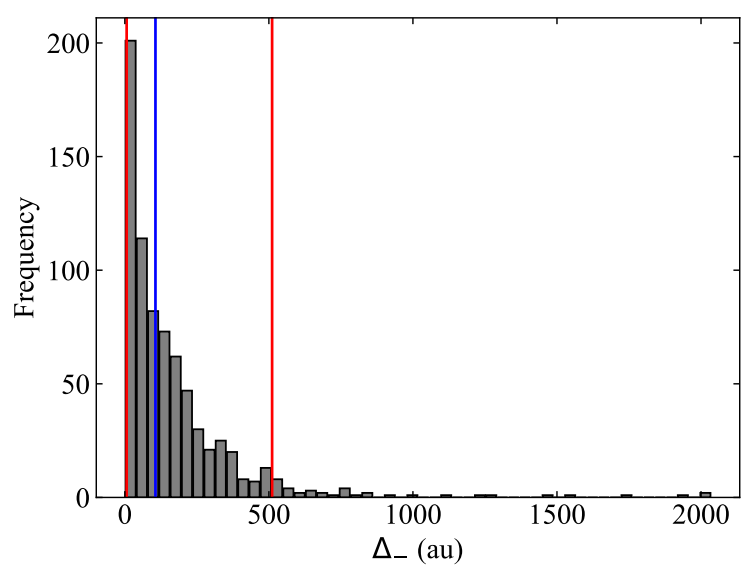

Figure 4. Distribution of mutual nodal distances for the descending nodes of the sample of 39 ETNOs. The median is shown in blue and the 5th and 95th percentiles in red.

(consistent with the asymmetry pointed out above), the two lowest values are for mutual ascending nodes. Out of 39 ETNOs, 41 per cent (16) have at least one mutual nodal distance smaller than $1.450 \mathrm{au}$. The existence of a subset of pairs with statistically sig- 


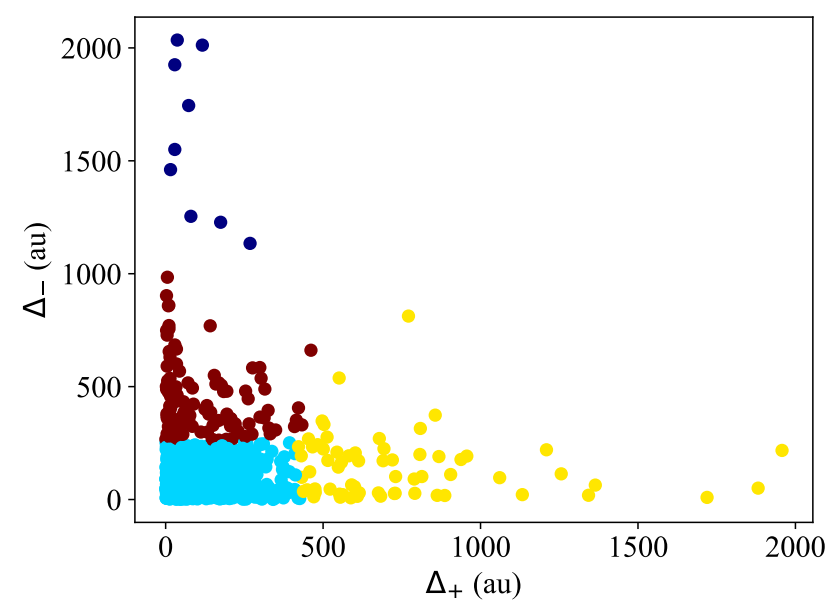

Figure 5. Colour-coded clusters generated by the $k$-means ++ algorithm applied to the dataset made of 741 pairs of mutual nodal distances from the sample of 39 ETNOs.

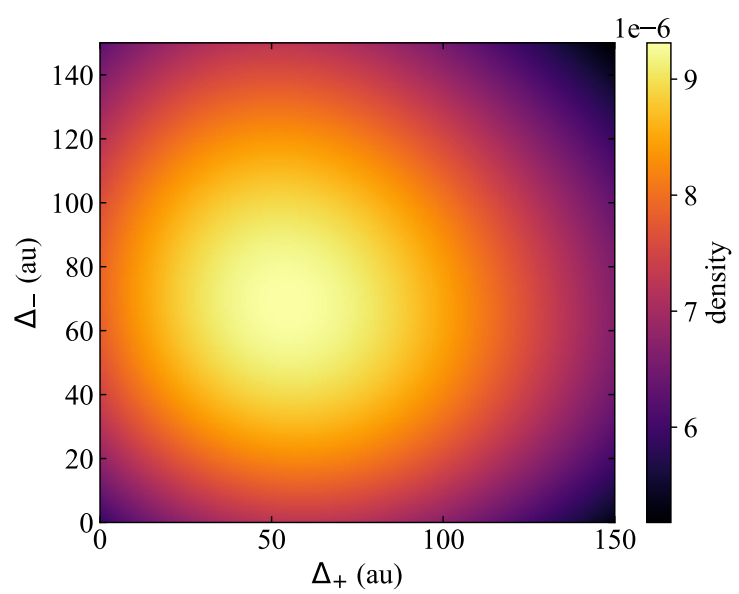

Figure 6. Gaussian kernel density estimation of the same data shown in Fig. 5 but focusing in the subsample with the smallest values of $\Delta_{ \pm}$(the region in dark blue in Fig. 5).

nificant small values of the mutual nodal distance suggests that the orbits of some known ETNOs are far from being dynamically uncorrelated. In sharp contrast, there are no pairs with both $\Delta_{ \pm}$below $5 \mathrm{au}$ and Fig. 6 shows that most pairs have $\left(\Delta_{+}, \Delta_{-}\right)$around (60 au, $70 \mathrm{au}$ ), well beyond the small values of the perhaps dynamically correlated pairs.

The ETNO pair with the lowest node separation consists of $148209\left(2000 \mathrm{CR}_{105}\right)$ and $505478\left(2013 \mathrm{UT}_{15}\right)$ with $\Delta_{+}=$ $0.23_{-0.16}^{+0.25}$ au (median and 16th and 84th percentiles, the median value is well below the 1 st percentile of the distribution) that are part of two different clusters ( 0 and 2, respectively) as shown in Table 1 . The second lowest value is found for the pair made of 496315 $\left(2013 \mathrm{GP}_{136}\right)$ and $2015 \mathrm{KG}_{163}$ with $\Delta_{+}=0.44_{-0.31}^{+0.43}$ au that again are in different clusters ( 0 and 1 , respectively). The pair consisting of $2002 \mathrm{~GB}_{32}$ and $2013 \mathrm{FT}_{28}$ has the third lowest mutual nodal distance with $\Delta_{-}=0.48_{-0.31}^{+0.36}$ au, and once more the objects are part of two different clusters ( 2 and 1, respectively). The pair consisting of $2016 \mathrm{QU}_{89}$ and $2016 \mathrm{SG}_{58}$ has the fourth lowest mutual nodal distance with $\Delta_{+}=0.62 \pm 0.13 \mathrm{au}$, although this time the objects are part of the same cluster, \#2. We would like to note that 148209 is part of two relevant pairs, but it is not the only ETNO being part of two or more pairs with low mutual nodal distances, $2013 \mathrm{SL}_{102}$ is also part of two and 474640 Alicanto $\left(2004 \mathrm{VN}_{112}\right), 505478$, and $2016 \mathrm{SG}_{58}$ are part of three. ETNOs 148209 and $2016 \mathrm{SG}_{58}$ are part of pairs with both $\Delta_{+}$and $\Delta_{-}$below 1.450 au. Column $X$ in Table 1 shows the number of pairs with one mutual nodal distance under 1.450 au for each known ETNO.

Our geometrical approach indicates that unless a protective mechanism is in place (perhaps in the form of mean-motion or secular resonances but see the comments in Section 1) and considering the uncertainties in the orbit determinations, close encounters at less than $10^{4} \mathrm{~km}$ (at the 5th percentile of the distribution of mutual nodal distances for some pairs, for example 148209 and 505478) may be possible and some of these objects have sizes of hundreds of $\mathrm{km}$ so approaches within 1 Hill radius of each other are theoretically achievable. These results open the door to actual collisions at relatively high speeds at perihelion and at aphelion as many known objects have the ascending nodes close to perihelion and the descending ones near aphelion. Whether this may actually be happening or not is difficult to assess because it implies making assumptions on how the Solar system is structured beyond $100 \mathrm{au}$ from the Sun. If there are no perturbers to keep these ETNOs at safe distances from each other, our analysis suggests that collisions may be taking place. Collisional families are not unheard of in the regular trans-Neptunian space, though. The first bona fide asteroid family identified in the outer Solar system was the one associated with dwarf planet Haumea (Brown et al. 2007) although a candidate collisional family had already been proposed by Chiang (2002) and later confirmed by de la Fuente Marcos \& de la Fuente Marcos (2018b) who found four new possible collisional families of TNOs and a number of unbound TNOs that may have a common origin. The subject of finding collisional families of TNOs has been studied by Chiang et al. (2003b) and Marcus et al. (2011).

\section{POLES AND PERIHELIA}

Here, we focus on the distributions of angular separations between orbital poles, $\alpha_{\mathrm{p}}$, and perihelia, $\alpha_{q}$, of the 39 ETNOs studied in the previous section. The orbital pole is the intersection between the celestial sphere and a hypothetical axis perpendicular to the plane of the orbit under study. The direction of perihelia is the intersection between the celestial sphere and a hypothetical line that goes from the focus of the orbit towards the point where the orbit under study reaches perihelion. Poles and perihelia are computed as described in Appendix B. Discarding $2020 \mathrm{KV}_{11}$ again, we have computed 741 pairs of $\left(\alpha_{q}, \alpha_{\mathrm{p}}\right)$ values. The results for each pair come from a set of $10^{4}$ pairs of virtual ETNOs as described in Appendix B. The distribution of angular distances between pairs of orbital poles is shown in Fig. 9 and that of the angular distances between pairs of perihelia is displayed in Fig. 10. These distributions have been computed using the data in Table 1 and considering the uncertainties in the orbit determinations as described in Appendixes A and B.

Applying the $k$-means++ algorithm to the $\left(\alpha_{q}, \alpha_{\mathrm{p}}\right)$ dataset as described in Section 3, we obtained Fig. 11 that displays three clusters, not four. The values of poles and perihelia provide the orientation of the orbit in space and therefore they are directly affected by the observational biases pointed out in Section 1. Nonetheless, 


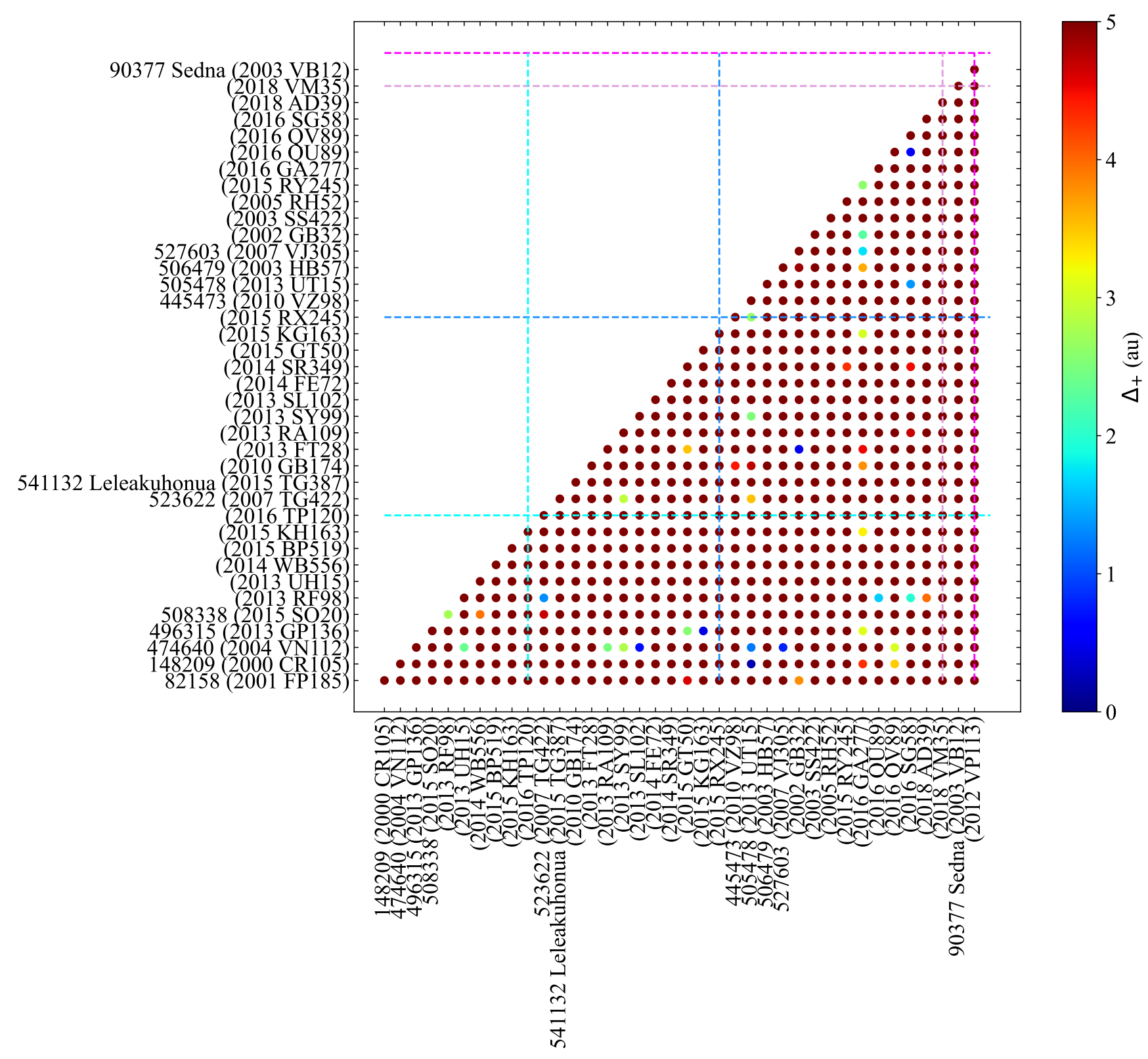

Figure 7. Colour-coded mutual ascending nodal distances for all the 741 pairs. As we are focusing on unusually low values of the mutual nodal distances, only the range $0-5$ au is shown in detail. Values of $\Delta_{ \pm}>5$ au appear in brown. The ETNOs have been sorted as they appear in Table 1 and the statistically significant clusters identified in Section 3.2 are indicated using dashed horizontal and vertical lines of colours consistent with those in Fig. 2, central panels.

the distributions in Figs 9 and 10 show that a number of pairs have small values of $\alpha_{q}$ and $\alpha_{\mathrm{p}}$. As we did in the previous section in order to identify severe outliers, we use the 1 st percentile of the distribution that is $7.59 \pm 0.02$ for $\alpha_{q}$ and $3.260289 \pm 0.000011$ for $\alpha_{p}$ (the 5 th percentile values are $15^{\circ} .19$ and 6.45 , respectively).

There are no pairs with both angular separations under the respective 1st percentile threshold, but there is one pair very close to it: $2013 \mathrm{FT}_{28}$ and $2015 \mathrm{KG}_{163}$, both from cluster 1, with $\alpha_{q}=$ $7.58_{-0.10}^{+0.11}$ and $\alpha_{p}=3.402 \pm 0.002$ (median and 16th and 84th percentiles). The pair consisting of $2010 \mathrm{~GB}_{174}$ (from cluster 1) and $2016 \mathrm{TP}_{120}$ (from cluster 0 ) has the lowest value of $\alpha_{q}=0.43^{+0^{\circ} .25}$ but $\alpha_{p}=11.219$ and the one including $523622\left(2007 \mathrm{TG}_{422}\right)^{-0.10}$ and $2016 \mathrm{GA}_{277}$ (from clusters 1 and 2, respectively) has the lowest value of $\alpha_{p}=0.8260^{+0.0004}$ but $\alpha_{q}=107.2$. In general, the orbits with the closest orientations in space tend to be part of different clusters. Some of the pairs following nearly intersecting orbits as discussed in the previous section also have similar orientations in space. The pair consisting of $148209\left(2000 \mathrm{CR}_{105}\right)$ and 505478 $\left(2013 \mathrm{UT}_{15}\right)$-from clusters 0 and 2 , respectively - that has the lowest node separation also has $\alpha_{q}=6.26 \pm 0.02$. Figure 12 shows that most pairs have $\left(\alpha_{q}, \alpha_{p}\right)$ around $\left(35^{\circ}, 20^{\circ}\right)$, well beyond the small values of the probably correlated pairs.

Another quantity of possible interest is the time of perihelion passage, $\tau_{q}$, not shown in Table 1 but whose distribution of differences is shown in Fig. 13. In this case, the 5th percentile value is $2.7 \mathrm{yr}$ and the 1 st percentile is $0.7 \mathrm{yr}$. The pair with the lowest value 


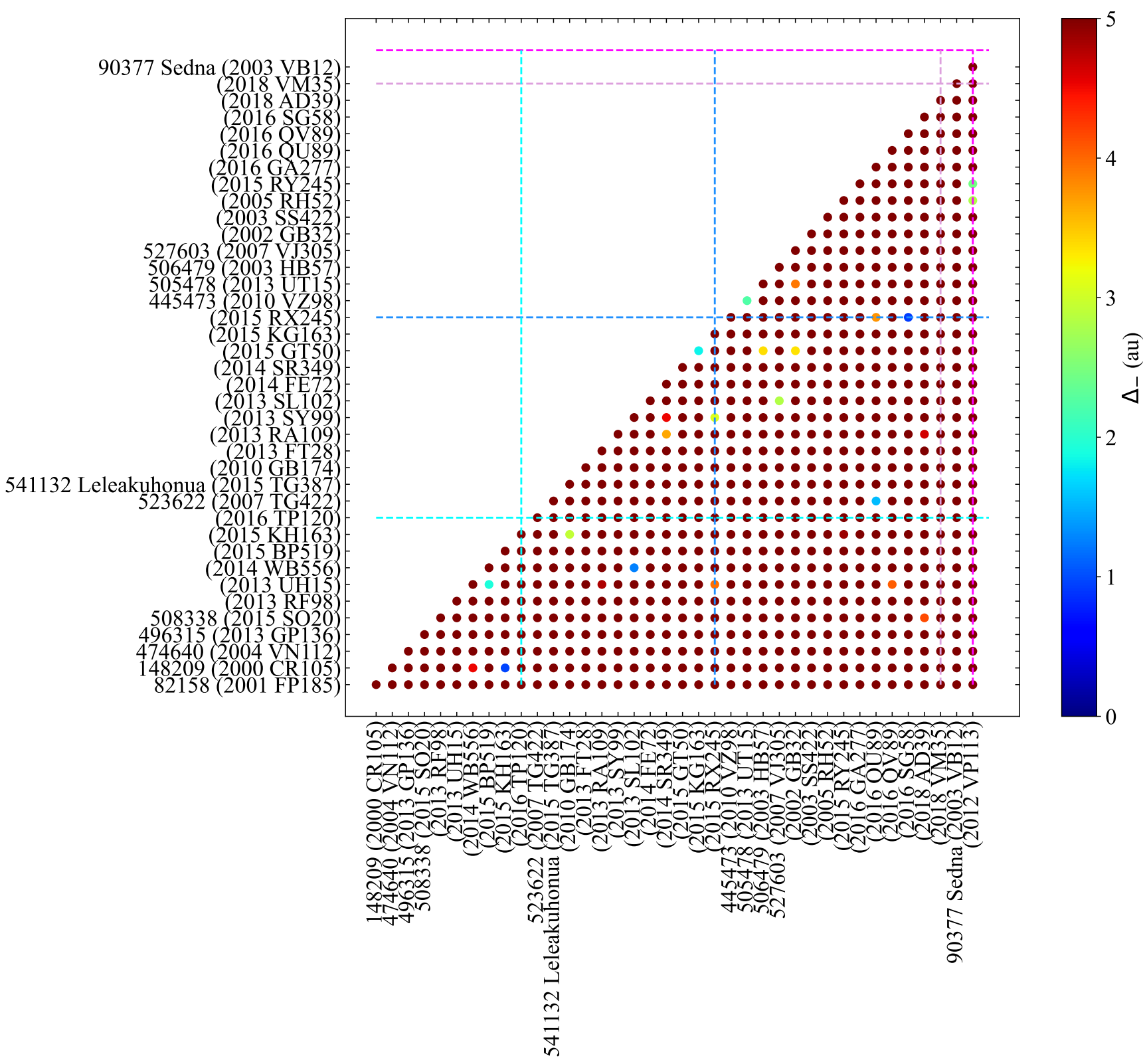

Figure 8. As Fig. 7 but for the descending nodes.

of $\Delta \tau_{q}$ is the one made of $523622\left(2007 \mathrm{TG}_{422}\right)$ of cluster 1 and $527603\left(2007 \mathrm{VJ}_{305}\right)$ of cluster 2 with $\Delta \tau_{q}=0.2020 \pm 0.0005 \mathrm{yr}$. The second lowest is for $2013 \mathrm{SY}_{99}$ of cluster 1 and $2015 \mathrm{KH}_{163}$ of cluster 0 with $\Delta \tau_{q}=0.21 \pm 0.03 \mathrm{yr}$. The pair with the third lowest value is the one made of 474640 Alicanto $\left(2004 \mathrm{VN}_{112}\right)$ of cluster 0 and $2013 \mathrm{RF}_{98}$ also from cluster 0 with $\Delta \tau_{q}=0.23_{-0.16}^{+0.23} \mathrm{yr}$. Such low values could be anecdotal as no pairs have very small differences in time of perihelion passage and also very low mutual nodal distances and/or very close angular separations $\alpha_{q}$ or $\alpha_{p}$.

\section{DISCUSSION}

We have performed detailed statistical analyses of a snapshot in orbital parameter space of the known ETNOs, a group of objects whose study may help in understanding how the Solar system is structured beyond $100 \mathrm{au}$ from the Sun. Our analyses do not make any assumptions on how the ETNOs came into existence or how they may be evolving dynamically, they only extract facts associated with this group of objects. It is now time to explore the implications of our findings.

The main question initially addressed in this work was that of the underlying level of clustering within the ETNOs. Are there any real clusters or the observed distribution is compatible with an uniform arrangement? This key issue has been previously studied by considering the orbital parameters separately and the results of those analyses have not been conclusive because of the observational bias discussed in Section 1. In order to avoid or at least minimize this problem, we have applied a novel approach by estimating the level of clustering in barycentric $\left(q_{\mathrm{b}}, e_{\mathrm{b}}, i_{\mathrm{b}}\right)$ space with 


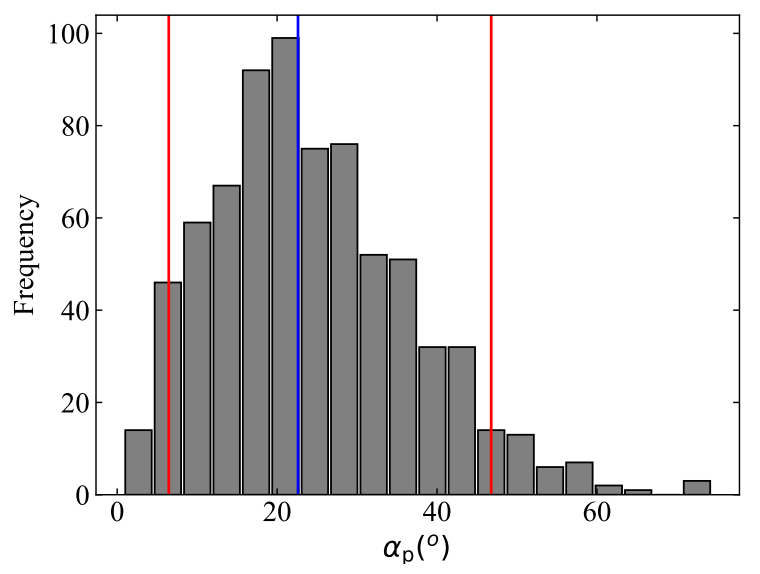

Figure 9. Distribution of angular distances between pairs of orbital poles of the sample of 39 ETNOs. The median is shown in blue and the 5th and 95th percentiles in red.

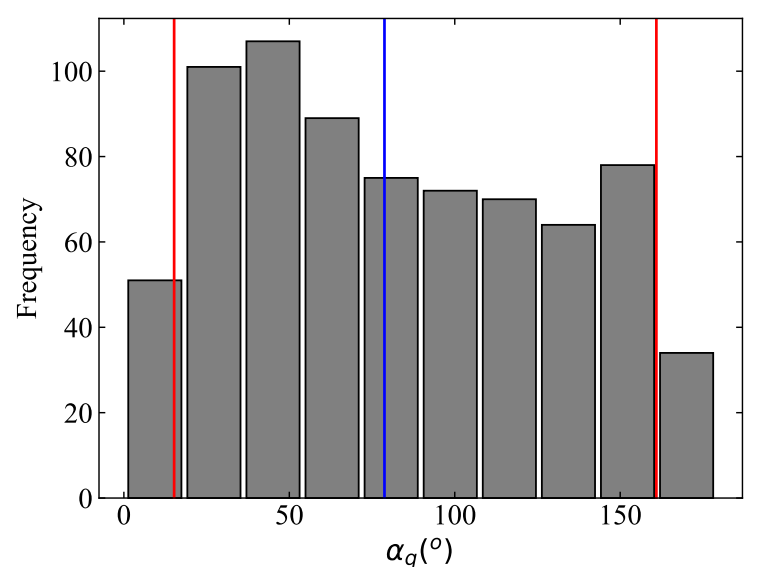

Figure 10. Distribution of angular distances between pairs of perihelia (its projected direction) of the sample of 39 ETNOs. The median is shown in blue and the 5 th and 95 th percentiles in red.

the $k$-means++ algorithm. Instead of focusing on one-dimensional clustering, we have applied the algorithm to a three-dimensional dataset. It may have been five-dimensional but we decided to leave the other two angular elements out of the analysis due to the bias concerns discussed in Section 1. The results of this analysis uncover four statistically significant clusters. This is not surprising when considering the case of the regular TNOs that consist of four or more dynamical classes. Therefore and on strictly mathematical grounds, we tentatively consider the answer to the original question as positive: there may be true clustering, the known ETNOs may not represent a single, monolithic population but the mixture of several, probably four, populations. On the other hand, the dynamical classes may be interacting and in this context, we may have to speak of dynamically active and dynamically inert ETNOs (see below, Section 6.1).

The actual statistical significance of our results cannot be properly assessed without considering a simplified model for the

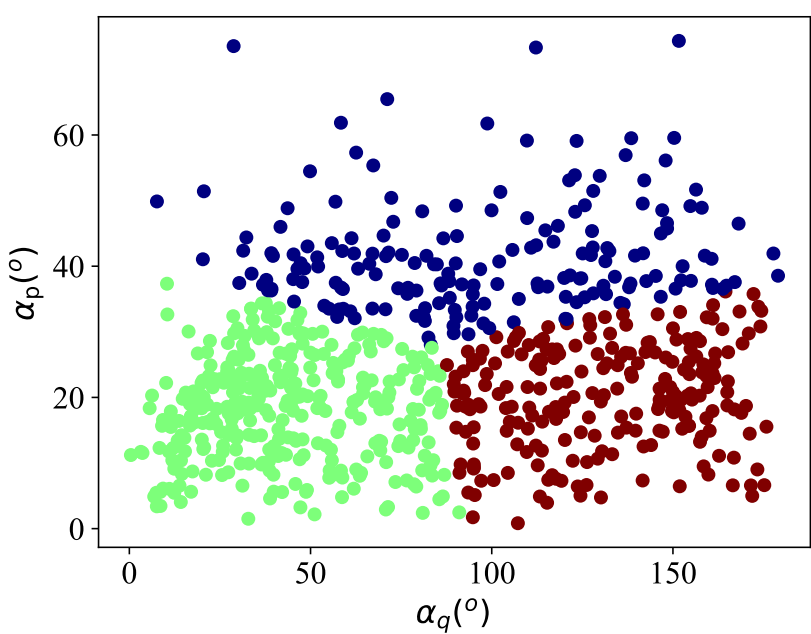

Figure 11. Colour-coded clusters generated by the $k$-means ++ algorithm applied to the dataset made of 741 pairs of $\left(\alpha_{q}, \alpha_{p}\right)$ from the sample of 39 ETNOs.

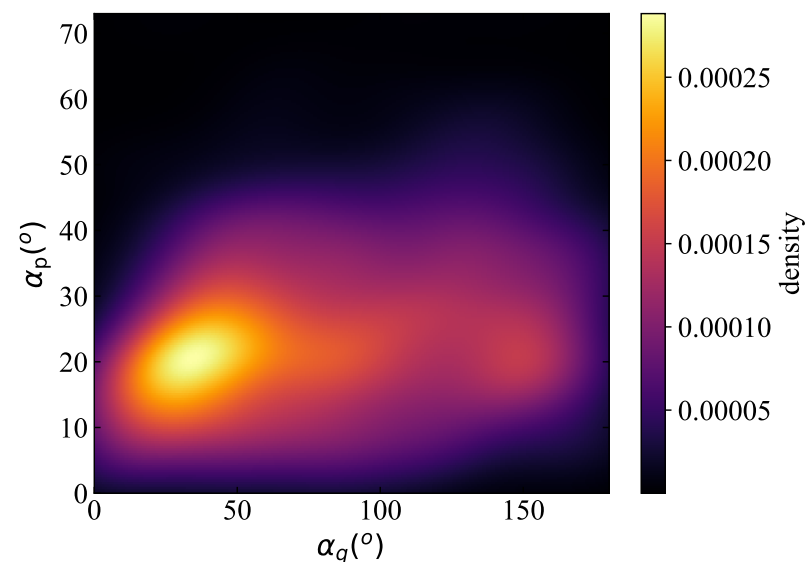

Figure 12. Gaussian kernel density estimation of the same data shown in Figs 9 and 10.

scattered disc (see below, Sections 6.2 and 6.3). Results from an unbiased model can then be compared with those coming from real ETNOs that are affected by the observational biases pointed out in Section 1. In the following, we have generated a synthetic sample consistent with results obtained by Brown $(2001,2017)$ and performed the same analysis carried out in Sections 4 and 5 on the resulting data. This, together with a shuffled version of the actual data serve as reference to estimate the statistical significance of our results. In a shuffled sample and by randomly reassigning the values of the orbital elements of the ETNOs, we preserve the original distributions of the parameters, but remove any possible correlations that may have been induced by hypothetical mean-motion or secular resonances, close encounters with massive perturbers or in response to external perturbations. 


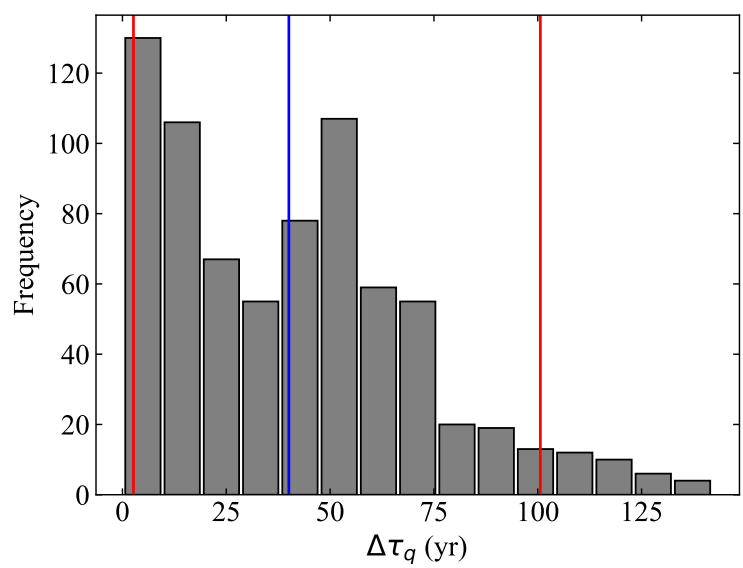

Figure 13. Distribution of differences in time of perihelion passage. The median is shown in blue and the 5th and 95th percentiles in red.

\subsection{Interacting populations: dynamically active versus inert ETNOs}

In Section 4, we have explored how the members of the candidate clusters interact within their own cluster and with members of other clusters. Two prograde Keplerian trajectories with a common focus can interact only in the vicinity of their mutual nodes, where the orbits are closest to each other. We would like to remark that our geometrical approach leaves out of the analysis the current position of the object in its orbit that is controlled by a sixth parameter, the mean anomaly, $M$. We believe that this additional parameter cannot provide significant information in the case of the ETNOs as they are all discovered at perihelion or very near it, so $M$ is always close to $0^{\circ}$ or $360^{\circ}$ for the current sample: the actual intervals are $\left(0.39,12^{\circ} .44\right)$ and $\left(348^{\circ} .07,359^{\circ} 97\right)$. The analysis presented in Section 4 shows that the nodal distances organize themselves into four clusters that we consider consistent with the four clusters found in barycentric $\left(q_{\mathrm{b}}, e_{\mathrm{b}}, i_{\mathrm{b}}\right)$ space.

The analysis also uncovers that out of 39 ETNOs, 16 or 41 per cent have at least one mutual nodal distance smaller than 1.45 au that is the 1st percentile of the distribution. We call these objects dynamically active ETNOs and they are identified in Table 1 by a value of $X>0$, the number of pairs with at least one mutual nodal distance smaller than 1.45 au in which they are members; dynamically inert ETNOs have $X=0$ in Table 1 . We believe that this result is quite remarkable when considering the large size of the orbits studied here. It is difficult to attribute this finding to mere coincidence and some processes should be operating in the background to lead to median values of the mutual nodal separation under 0.5 au in pairs such as $148209\left(2000 \mathrm{CR}_{105}\right)$ and $505478\left(2013 \mathrm{UT}_{15}\right)$. The fraction of dynamically active ETNOs considering the simple scattered disc model discussed in the next section is 54 per cent and the one from an experiment with shuffled orbital parameters is also 54 per cent. This result, obtained randomly sampling 39 orbits $10^{4}$ times, strongly suggests that, in this regard, shuffling the observed orbital parameters is equivalent to considering the simple scattered disc model.

In addition, some pairs of orbits (for example, those of 148209 and 505478) have non-negligible probabilities (small but $>5$ per cent) of approaching each other under a few thousand kilometers when uncertainties are factored in. This fact can be signalling the existence of collisions or of mechanisms to avoid them such as mean-motion or secular resonances. If we study the dynamically active fraction in the samples of $10^{4}$ synthetic ETNOs (so 49995000 pairs), we find that 100 per cent of the objects have at least one mutual nodal distance smaller than $1.45 \mathrm{au}$; in fact, each object is part of over 200 pairs in which one nodal distance is smaller than $1.45 \mathrm{au}$. This result together with the previous one of an active fraction of 54 per cent when samples of 39 objects are considered suggests that there is a probable excess of dynamically inert ETNOs.

Within the context of the simple scattered disc model, physical collisions or orbit-changing close encounters (if massive ETNOs are involved) could be ubiquitous unless protective mechanisms are in place. Close encounters and collisions in the transNeptunian or Kuiper belt are relatively well understood (see e.g. Dell'Oro et al. 2013; Bromley \& Kenyon 2020; Kenyon \& Bromley 2020; Abedin et al. 2021). Collisional families are known to populate regular TNO space that is also affected by orbital resonances with small integer ratios and secular resonances like the so-called von Zeipel-Lidov-Kozai oscillation (von Zeipel 1910; Lidov 1962; Kozai 1962; Ito \& Ohtsuka 2019). Resonances are driven by massive perturbers that, in the case of regular TNOs, have Neptune as main driver and the other giant planets as secondary perturbers. Close encounters with a massive perturber can also produce pairs that resemble those observed via binary stripping (de la Fuente Marcos, de la Fuente Marcos \& Aarseth 2017).

No massive perturbers are known to exist in ETNO space, but the exploration of this vast region is just beginning (see e.g. Holman et al. 2018; Perdelwitz, Völschow \& Müller 2018; Kaib et al. 2019; Sheppard, Trujillo \& Tholen 2019; Bernardinelli et al. 2020b; Rice \& Laughlin 2020; Naess et al. 2021). Trujillo \& Sheppard (2014) argued that the orbital organization of the few ETNOs known in 2014 was indicative of the presence of one hidden massive perturber at hundreds of au from the Sun. However, de la Fuente Marcos \& de la Fuente Marcos (2014) and de la Fuente Marcos, de la Fuente Marcos \& Aarseth (2015) contended that the available data at the time were better explained if more than one massive perturber was orbiting the Sun well beyond Pluto. The size of the ETNO dataset was still limited to less than a dozen objects when Batygin \& Brown (2016) presented the so-called Planet 9 hypothesis as an explanation for the orbital architecture of the known ETNOs. The discovery of additional objects led to modifications of the original hypothesis.

The latest incarnation of the Planet 9 hypothesis (Batygin et al. 2019) predicts the existence of a planet with a mass in the range 5-10 $M_{\oplus}$, following an orbit with a value of the semimajor axis in the range of 400-800 au, eccentricity in the range of $0.2-0.5$, and inclination in the interval between $\left(15^{\circ}, 25^{\circ}\right)$. However, Fienga et al. (2020) used the INPOP19a planetary ephemerides that include Jupiter-updated positions by the Juno mission and a reanalysis of Cassini observations to conclude that if Planet 9 exists, it cannot be closer than $500 \mathrm{au}$, if it has a mass of $5 M_{\oplus}$, and no closer than $650 \mathrm{au}$, if it has a mass of $10 M_{\oplus}$. A recent study using Monte Carlo random search techniques (de la Fuente Marcos \& de la Fuente Marcos 2021) has found that if the high eccentricities of the known ETNOs are the result of relatively recent flybys with putative planets, these perturbers might not be located farther than 600 au and they have to follow moderately eccentric and inclined orbits to be capable of experiencing close encounters with multiple known ETNOs. This result places the perturbers in the so-called inert Oort Cloud proposed by Saillenfest et al. (2019). Planetary bodies may have been inserted there early in the history of the Solar system via 
planetary scattering (see e.g. Bromley \& Kenyon 2016; Bailey \& Fabrycky 2019) or, less likely, formed in situ (see e.g. Kenyon \& Bromley 2015, 2016).

Focusing on how the ETNOs with one mutual nodal distance smaller than 1.45 au are distributed across the clusters in Table 1 (those with $X>0)$, there are six from cluster $0(6 / 11,54.5$ per cent), five from cluster $1(5 / 12,41.7$ per cent), five from cluster $2(5 / 15,33.3$ per cent), and none from cluster 3 , Sednoids $(0 / 2$, 0 per cent). Six pairs include a member of cluster 1 , ten pairs include members of cluster 0 , nine pairs include members of cluster 2 , and there are no pairs including members of cluster 3 . Within each cluster there are dynamically inert members whose orbits appear to be well detached from all the other orbits and dynamically active members that seem to have the theoretical capability of interacting with members of the same cluster (seldom) and of other clusters (more often) as seen in Figs 7 and 8. These dynamically active members include 148209 , 474640 Alicanto (2004 $\mathrm{VN}_{112}$ ), 505478, $2013 \mathrm{SL}_{102}$ and $2016 \mathrm{SG}_{58}$. The cluster with the largest number of dynamically active members is 0 and the one with the smallest is 3; the smallest cluster, the Sednoids, does not have any dynamically active members.

The case of Alicanto and 2013 SL $_{102}$ is particularly indicative of the existence of some level of gravitational interaction because they have a short mutual ascending nodal distance, $\Delta_{+}=$ $0.66_{-0.36}^{+0.37} \mathrm{au}$, and a small difference in time of perihelion passage, $\Delta \tau_{q}=0.71 \pm 0.03 \mathrm{yr}$, therefore they may pass relatively close to each other within a relatively narrow time window as their orbits have nearly synchronized perihelion passages. As pointed out above, the 1st percentile of the distribution of differences in time of perihelion passage is $0.7 \mathrm{yr}$ so the value of $\Delta \tau_{q}$ for this pair is barely beyond the outlier boundary considered in this work. In sharp contrast, objects like Sedna could be considered as dynamically inert. It is unclear why do we have two distinct groups regarding mutual nodal distances, but this is unlikely to be accidental or due to observational biases. The cluster with the smallest number of dynamically active members is cluster 3 , it is also the one with the least members. The properties of cluster 1 make it compatible with an origin in the so-called inner Oort Cloud (Levison, Dones \& Duncan 2001) that was first proposed by Hills (1981).

When considering the dynamically active objects, if they are somehow related, it is unlikely that they formed during a gentle split (i.e. the relative speed at separation could have been low, slightly above the escape velocity from the surface of the most massive object) given the range of values of the various parameters discussed in the previous sections. A more plausible scenario may be that of a high speed collision with catastrophic effects producing two clouds of sizeable fragments moving along the original paths that eventually spread throughout the colliding orbits. In this scenario, two large fragments undergoing a flyby at a later time may have different surface properties and their relative velocity at the distance of closest approach could be close to the original impact speed as they come from the two original, likely unrelated impactors (for example, consider the case of 148209 and 505478). There are few spectroscopic results published on the surface composition of the ETNOs. The results in de León, de la Fuente Marcos $\&$ de la Fuente Marcos (2017) show that the spectral slopes of Alicanto and $2013 \mathrm{RF}_{98}$, both in cluster 0 and with one of the lowest values of $\Delta \tau_{q}$, are very close matches and compatible with the ones of $2002 \mathrm{~GB}_{32}$ and $506479\left(2003 \mathrm{HB}_{57}\right)$, both in cluster 2 . However, they are very different from that of Sedna that is the reference object in cluster 3 . The spectral slope could in principle be used to separate groups, but if the orbital evolution in ETNO space is chaotic
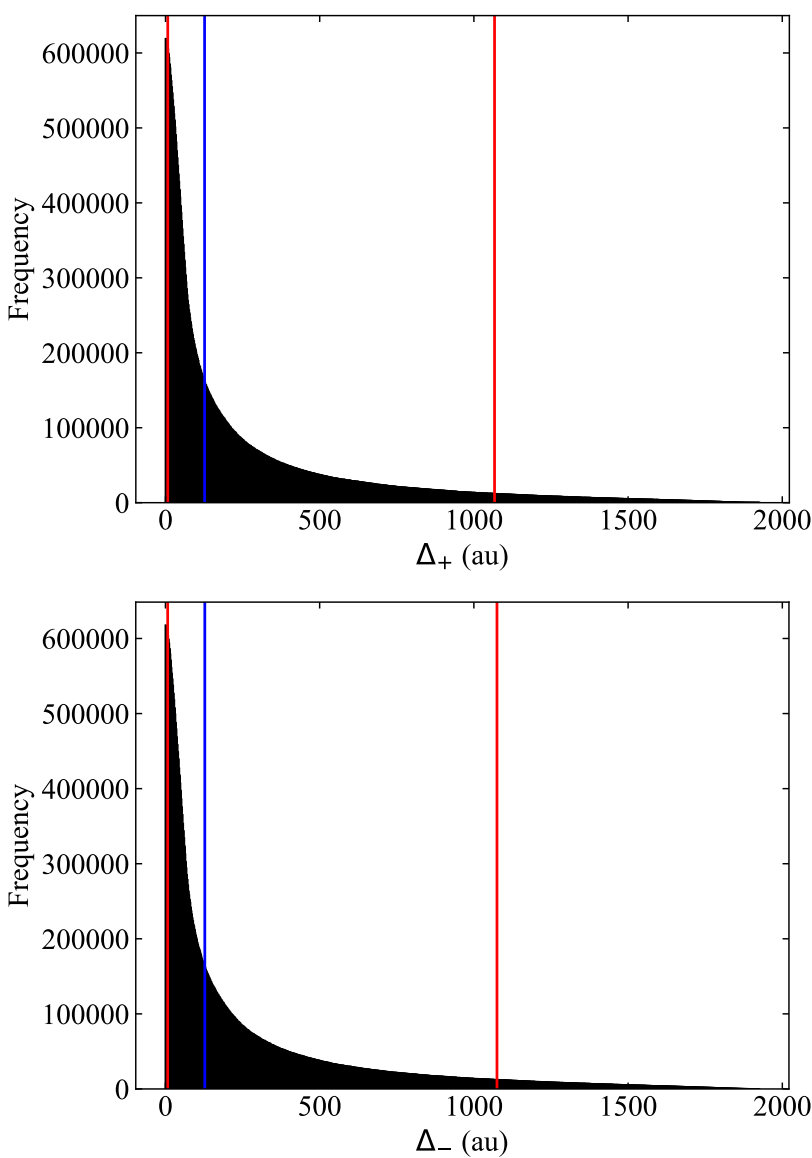

Figure 14. Distribution of mutual nodal distances of ascending nodes (top panel) and of descending nodes (bottom panel) of synthetic ETNOs. The median is shown in blue and the 5 th and 95 th percentiles in red.

due to the presence of some yet-undetected perturbers (or any other capable dynamical mechanism), true dynamical classes may host objects with different spectral slopes. Considering together spectral and orbital information may help in understanding better how the various objects could be related, but published spectral data on the ETNOs are still scarce.

\subsection{Statistical significance}

Let us consider a scattered disc model in which $a$ follows a uniform distribution in the interval $(150,1000)$ au, $q$ is also uniform in the interval $(30,100)$ au, the angular elements $\Omega$ and $\omega$ are drawn from a uniform distribution in the interval $\left(0^{\circ}, 360^{\circ}\right)$, and $i$ follows the so-called Brown distribution of inclinations (Brown 2001, 2017) in the interval $\left(0^{\circ}, 60^{\circ}\right)$ as described in Appendix C. After analyzing, as pointed out above, 10 instances of $10^{4}$ synthetic ETNOs each (or 49995000 pairs per instance), we found that the 1st percentile of $\Delta_{+}$ is $1.460 \pm 0.009 \mathrm{au}$ and the one for $\Delta_{-}$is $1.450 \pm 0.009 \mathrm{au}$. Therefore and under the assumption of a uniform scattered disc model with a Brown distribution of inclinations, we expect a symmetrical distribution of mutual nodal distances; in other words, the median and percentiles of the mutual ascending and descending nodal distances must be identical (see Fig. 14).

On the other hand, we observe that the values of the 1 st percentile of $\Delta_{+}$for the simple model and the one of the actual data are 


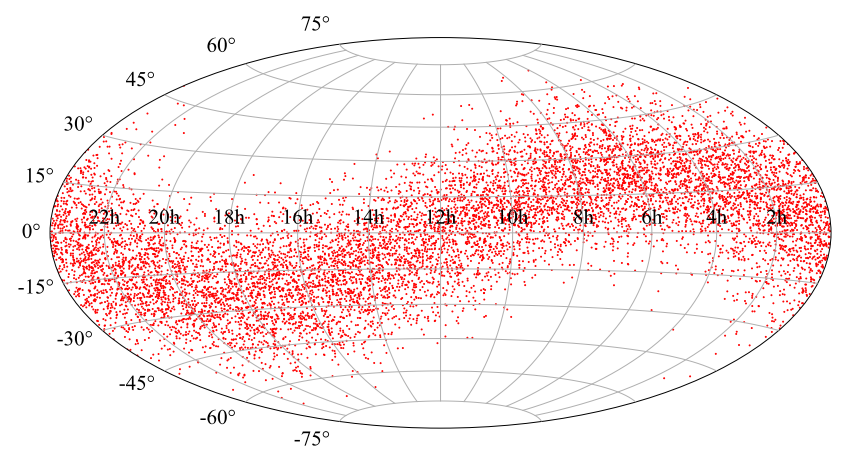

Figure 15. Distribution of the missing synthetic ETNOs that may have produced pairs with $\Delta_{-}$under 2.335 au according to the model discussed in Section 6.1 .

virtually identical ( $1.1 \sigma$ difference, considering the model as reference). In sharp contrast and for $\Delta_{-}$, we find a difference of over $98 \sigma$ between the model and the real data. It is difficult to conclude that such a consistent match in $\Delta_{+}$could be the result of chance alone; the simple scattered disc model discussed above must, in some way, resemble the actual distributions of orbital elements of the ETNOs.

As for the strong asymmetry (model versus real data) found between the match in the 1st percentile of $\Delta_{+}$and the large difference in the 1st percentile of $\Delta_{-}$, the easiest explanation could be that it may be linked to the biases in the angular elements discussed above. This, however, has strong implications: the missing low values of the mutual descending nodal distances must correspond to pairs that have not been found by the various surveys contributing discoveries of this type of objects. Figure 15 shows the predicted locations at perihelia (where the known ETNOs have been found) of such missing objects (the ones with $\Delta_{-}<2.335 \mathrm{au}$ ) according to the model. They outline the ecliptic but in a rather random fashion so it is difficult to assume that the statistically significant asymmetry between the shortest mutual ascending and descending nodal distances present in the data could be the exclusive result of observational bias, other effects must be at work. In addition, the asymmetry persists when considering $10^{4}$ samples of 39 orbits obtained by shuffling the orbital parameters in Table 1 . The observational biases discussed in the literature (see Section 1) affect the angular elements but not $a$ or $e$ and, therefore, not $q$. The asymmetry might have arisen in response to external perturbations.

Continuing with the predictions from the simple scattered disc model discussed above, the pair of ETNOs in cluster 2 made of $505478\left(2013 \mathrm{UT}_{15}\right)$ and $2016 \mathrm{SG}_{58}$ has $\Delta_{+}=1.35_{-0.95}^{+1.37}$ au at $339 \mathrm{au}$ from the barycentre of the Solar system. The probability of finding a pair of synthetic ETNOs with $\Delta_{+}<1.450$ au beyond 300 au is $0.000205 \pm 0.000005$ (the odds are nearly 1 in 5000 so one has to have 5000 pairs, not 741 , to be able to find such a distant close nodes). Therefore, if the observed ETNOs come from a distribution similar to the simple scattered disc model used in this section, this pair is truly unusual and should not have been observed in a sample of just 39 objects (see the distribution of distances to the nodes in Fig. 16). If we randomly sample 39 orbits $10^{4}$ times from the simple scattered disc model and by shuffling the orbital parameters, we obtain a consistent result: that no pairs with close mutual nodes must exist beyond 300 au and that the 99th percentile of the distribution of barycentric nodal distances is $\sim 110$ au.

As for the orientations in space of the orbits, the 1st percentile of $\alpha_{q}$ from the model is $7.70 \pm 0.02$ and $2.9 \pm 0.2$ for $\alpha_{p}$. In this case,
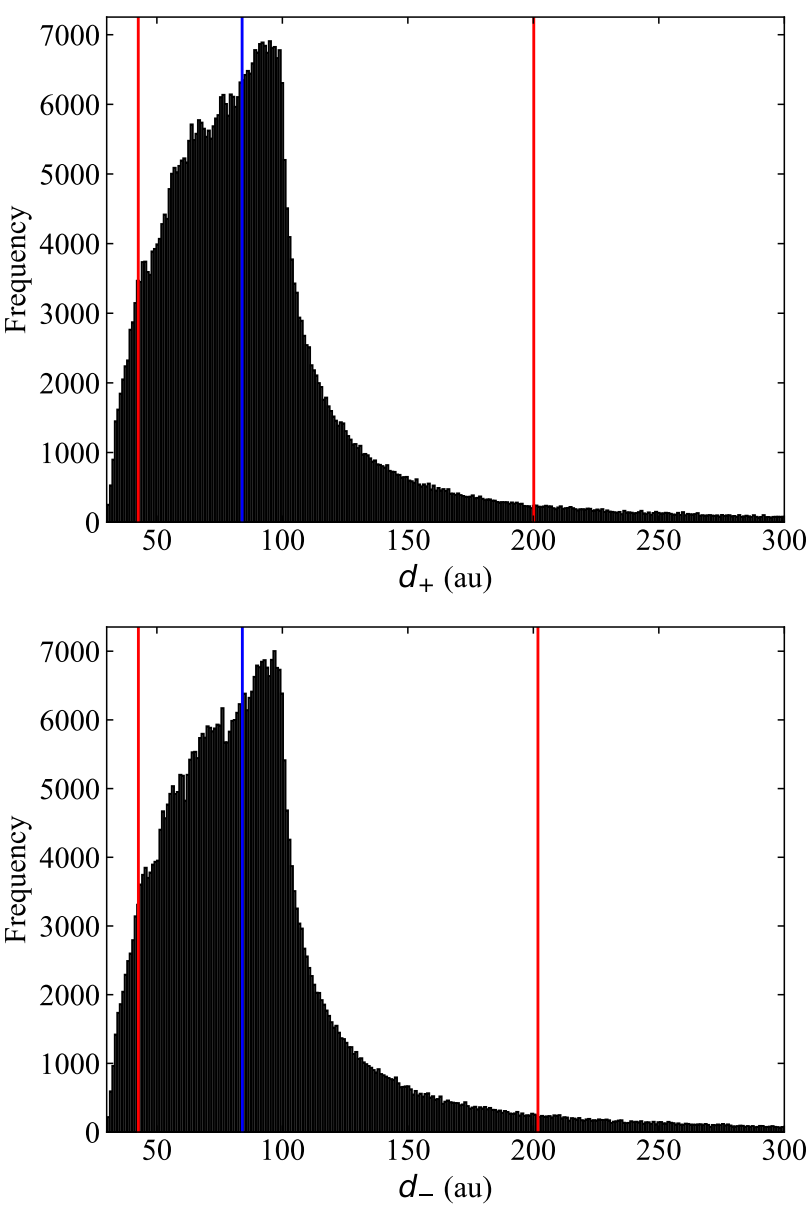

Figure 16. Distribution of barycentric distances to mutual ascending nodes (top panel) and descending nodes (bottom panel) of synthetic ETNOs $\left(10^{4}\right)$. The median is shown in blue and the 5 th and 95 th percentiles in red.

the discrepancies between model an real data in the low-probability domain are less pronounced ( $5.5 \sigma$ and $1.8 \sigma$, respectively). There is however an obvious issue when comparing the observational distribution in $\alpha_{q}$ (see Figs 9-12) and that of the synthetic ETNOs (see Fig. 17, bottom panel): the second maximum at about $145^{\circ}$ is missing from the distribution. As the distributions of $\alpha_{q}$ and $\alpha_{p}$ only depend on the angular elements and these are affected by observational biases, the most plausible explanation for the missing maximum is in the biases themselves.

In order to check for internal consistency, we have performed the analysis of $10^{6}$ samples of 39 ETNOs obtained by randomly scrambling or shuffling the orbital parameters in Table 1 and another set of $10^{6}$ samples of 39 synthetic ETNOs obtained as pointed out in this section. The results from both sets are virtually the same and we interpret this outcome as supportive of two conclusions: that the simple scattered disc model could be good enough as reference and that the known ETNOs are affected by hidden orbital correlations that may not be the exclusive result of observational biases.

\subsection{Caveats}

There are a number of caveats which concern the robustness of the statistical significance estimates computed in the previous sec- 

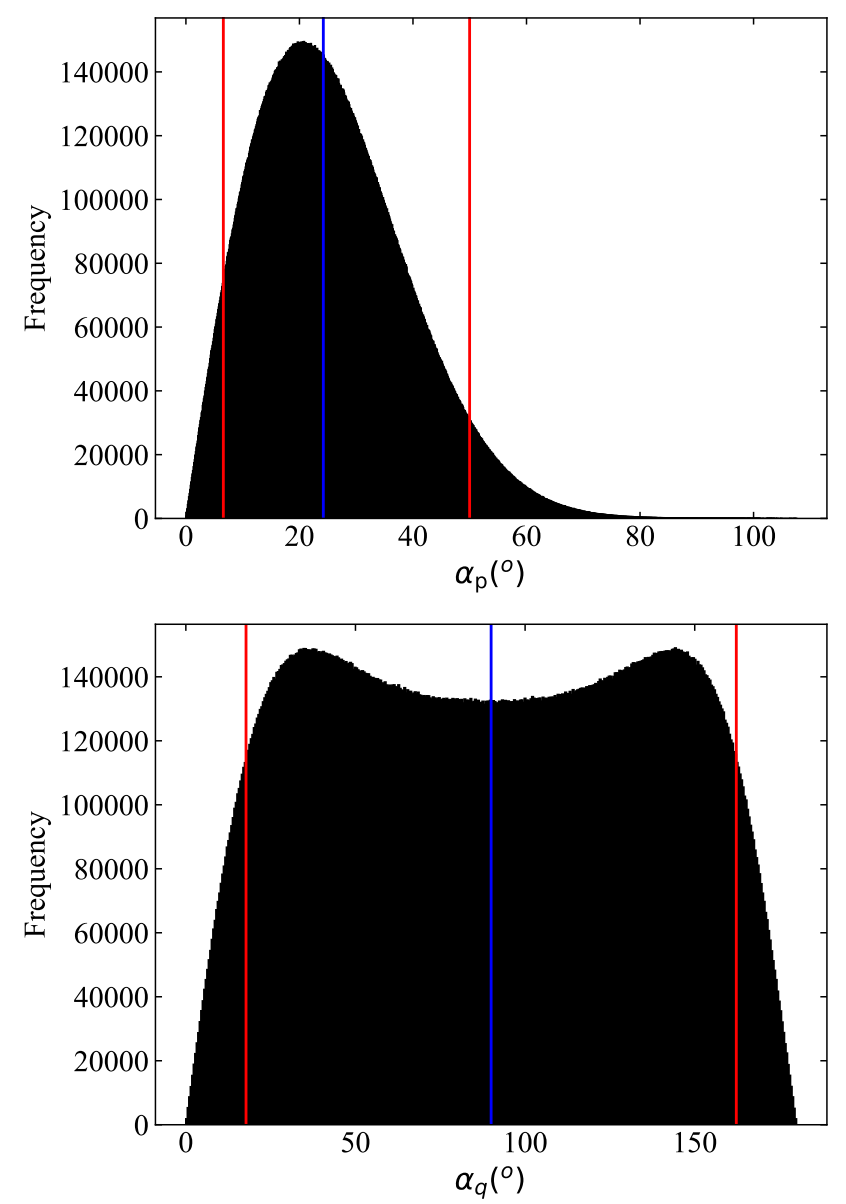

Figure 17. Distribution of angular distances between pairs of orbital poles (top panel) and of angular distances between pairs of perihelia (its projected direction, bottom panel) of synthetic ETNOs $\left(10^{4}\right)$. The median is shown in blue and the 5th and 95th percentiles in red.

tion. The simple scattered disc model discussed above is just one of many that may be used as a reference. Napier et al. (2021) argue for a model in which $a$ follows the distribution $N(a) \propto a^{0.7}, e$ is uniformly distributed in the interval $(0.69,0.999), i$ follows the Brown distribution as before, and the angular elements $\Omega$ and $\omega$ are also drawn from a uniform distribution in the interval $\left(0^{\circ}, 360^{\circ}\right)$. Under such model, the orientations in space of the orbits $\left(\alpha_{q}, \alpha_{p}\right)$, remain unchanged, but the distributions of $\Delta_{ \pm}$are quite different as the disc is significantly less concentrated than in the case discussed in the previous section (see Fig. 18). For this model (also $10^{4}$ synthetic ETNOs), the 1 st percentile of $\Delta_{+}$is 2.98 au and the one for $\Delta_{-}$is $2.97 \mathrm{au}$. The asymmetry pointed out above remains and now the unusually low values of $\Delta_{ \pm}$presented above are even more significant (less probable). The probability of finding a pair of synthetic ETNOs in this model with $\Delta_{+}<1.450$ au beyond $300 \mathrm{au}$ is $0.00047 \pm 0.00001$ that is still very low, although higher than the one obtained with the model discussed in the previous section.

The origin of the improbable orbits (in terms of correlations) observed and the statistically robust asymmetry present in the distribution of mutual nodal distances computed for the known ETNOs is open to discussion. Collisions and the effects of yet-to-bediscovered massive perturbers (see above) may have a role in explaining what is observed as well as stellar encounters and (less
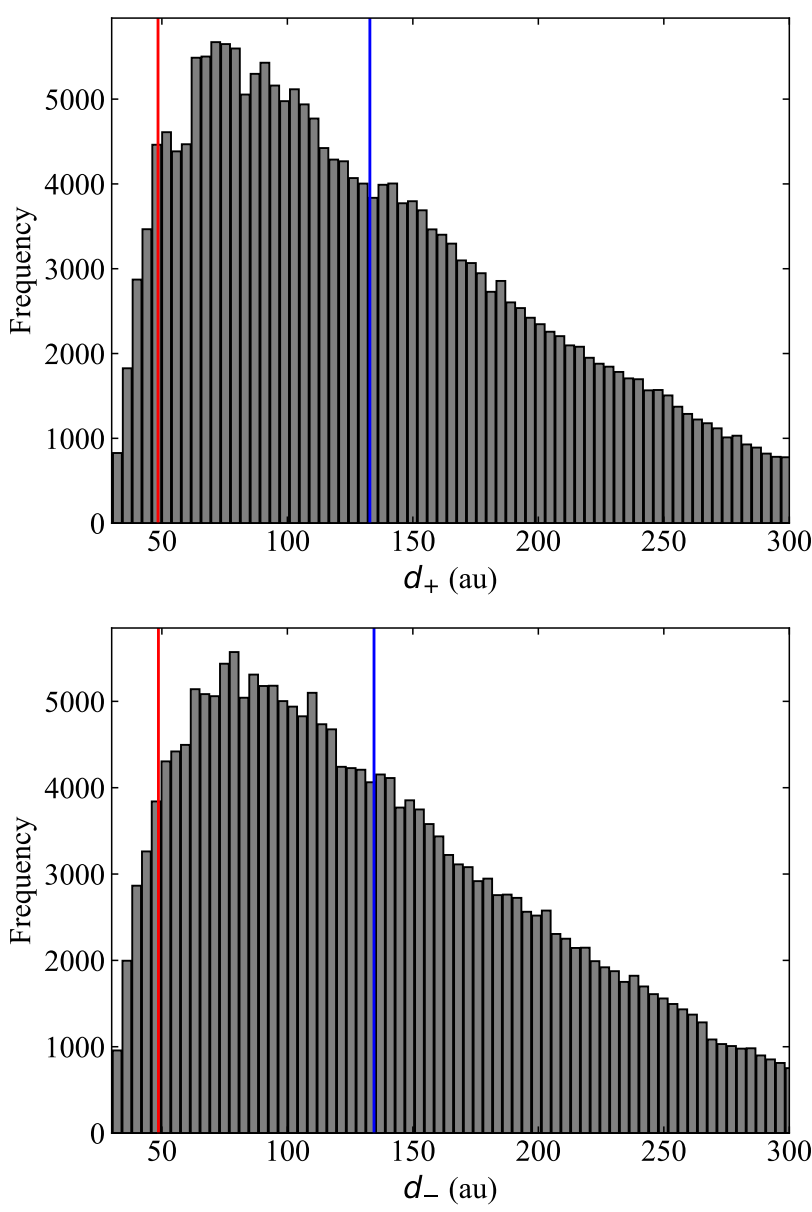

Figure 18. As Fig. 16 but for the model in Napier et al. (2021).

likely) the Galactic tide (see e.g. Pfalzner et al. 2018). The warped midplane of the trans-Neptunian belt discussed by Volk \& Malhotra (2017a,b) might also evidence past stellar encounters (see e.g. de la Fuente Marcos, de la Fuente Marcos \& Aarseth 2018; Moore, Li \& Adams 2020) and/or be linked to the asymmetry uncovered by our analyses.

\section{CONCLUSIONS}

In this paper, we have applied machine-learning techniques to explore the possible existence of clusterings or subgroups within the ETNO group of asteroids (those with $a>150$ au and $q>30 \mathrm{au}$ ). Then, we have investigated the presence of correlations using the distributions of the nodal distances and the poles and perihelia of the orbits within the context of the uncertainties of their barycentric orbit determinations. The main conclusions of our study are:

(i) We identify four statistically significant groups or clusters in $\left(q_{\mathrm{b}}, e_{\mathrm{b}}, i_{\mathrm{b}}\right)$ ETNO orbital parameter space by applying the $k$-means++ algorithm.

(ii) One of the statistically significant groups includes 90377 Sedna $\left(2003 \mathrm{VB}_{12}\right)$ and $2012 \mathrm{VP}_{113}$ and appears to be well dynamically detached from the rest of the ETNOs.

(iii) Another statistically significant group may be related to the inner Oort cloud and includes objects like 541132 
Leleakuhonua (2015 $\left.\mathrm{TG}_{387}\right), 2013 \mathrm{SY}_{99}, 2014 \mathrm{FE}_{72}$, and $2015 \mathrm{KG}_{163}$.

(iv) The distribution of mutual nodal distances shows a statistically significant asymmetry between the shortest mutual ascending and descending nodal distances. We argue that this statistically robust asymmetry is not due to observational bias but perhaps the result of external perturbations.

(v) Although most nodal distances are found in the range 60$70 \mathrm{au}$, out of 39 ETNOs, 16 or 41 per cent have at least one mutual nodal distance smaller than 1.45 au that is the 1 st percentile of the distribution. There are no pairs with both mutual nodal distances smaller than 5 au.

(vi) The peculiar pair of ETNOs made of 505478 (2013 UT 15 ) and $2016 \mathrm{SG}_{58}$ has a mutual ascending nodal distance of 1.35 au at 339 au from the Sun. We estimate that the probability of finding such a pair by chance is $0.000205 \pm 0.000005$.

(vii) The orientations in space of the orbits of the ETNOs show an asymmetric distribution of angular separations between perihelia that we tentatively attribute to observational bias.

(viii) Although most angular separations between orbital poles and perihelia are close to $30^{\circ}$, there are some pairs of ETNOs with similar values of poles and perihelia. The strongest outlier is the pair $2013 \mathrm{FT}_{28}-2015 \mathrm{KG}_{163}$.

(ix) The observed distributions of $\Omega, \omega$ and $\varpi=\Omega+\omega$ of the ETNOs appear to be compatible with those of a uniform or random sample.

(x) The inclination distribution of the known ETNOs seems to follow the law discussed by Brown $(2001,2017)$

The statistical significance of our results is unexpectedly robust, the simple scattered disc model discussed in Section 6 is able to reproduce relatively well the observed outlier limits for $\Delta_{+}, \alpha_{q}$ and $\alpha_{p}$ as well as the actual distributions in $\Delta_{+}$and $\alpha_{p}$; it fails, however, in reproducing the observed distributions of $\Delta_{-}$and $\alpha_{q}$. Observational bias may be regarded as the origin of the discrepancies in $\alpha_{q}$, but the $\Delta_{+}-\Delta_{-}$asymmetry is probably not due to observational bias. Our results show that one may have uniformity in $\Omega$ and $\omega$ within the extreme outer Solar system orbital parameter space as advocated by e.g. Shankman et al. (2017) or Bernardinelli et al. (2020a) or Napier et al. (2021) and still preserve tantalizing signals of present-day gravitational perturbations like the highly statistically significant asymmetry between the shortest mutual ascending and descending nodal distances identified here or the existence of at least one peculiar pair of ETNOs — 505478 and $2016 \mathrm{SG}_{58}$ with a small value of the mutual nodal distance and located beyond $300 \mathrm{au}$ from the barycentre of the Solar system.

\section{ACKNOWLEDGEMENTS}

We thank the referee for her/his constructive, actionable, and insightful reports that included very helpful suggestions regarding the presentation of this paper and the interpretation of our results, $\mathrm{J}$. Giorgini for extensive comments on JPL's SBDB and HORIZONS systems, S. J. Aarseth, J. de León, J. Licandro, A. Cabrera-Lavers, J.-M. Petit, M. T. Bannister, D. P. Whitmire, G. Carraro, E. Costa, D. Fabrycky, A. V. Tutukov, S. Mashchenko, S. Deen and J. Higley for comments on ETNOs, and A. I. Gómez de Castro for providing access to computing facilities. This work was partially supported by the Spanish 'Ministerio de Economía y Competitividad' (MINECO) under grant ESP2017-87813-R. In preparation of this paper, we made use of the NASA Astrophysics Data System and the MPC data server.

\section{DATA AVAILABILITY}

The data underlying this paper were accessed from JPL's SBDB (https://ssd.jpl.nasa.gov/sbdb.cgi). The derived data generated in this research will be shared on reasonable request to the corresponding author.

\section{REFERENCES}

Abedin A. Y. et al., 2021, AJ, 161, 195

Adams E. R., Gulbis A. A. S., Elliot J. L., Benecchi S. D., Buie M. W., Trilling D. E., Wasserman L. H., 2014, AJ, 148, 55

Arthur D., Vassilvitskii S., 2007, in Gabow H., ed., Proceedings of the eighteenth annual ACM-SIAM symposium on discrete algorithms, Society for Industrial and Applied Mathematics Philadelphia, PA, USA, p. 1027

Bailey N., Fabrycky D., 2019, AJ, 158, 94

Bannister M. T. et al., 2018, ApJS, 236, 18

Batygin K., Brown M. E., 2016, AJ, 151, 22

Batygin K., Brown M. E., 2021, ApJ, 910, L20

Batygin K., Adams F. C., Brown M. E., Becker J. C., 2019, Phys. Rev., 805, 1

Becker J. C. et al., 2018, AJ, 156, 81

Bernardinelli P. H. et al., 2020a, PSJ, 1, 28

Bernardinelli P. H. et al., 2020b, ApJS, 247, 32

Bernstein G. M., Trilling D. E., Allen R. L., Brown M. E., Holman M., Malhotra R., 2004, AJ, 128, 1364

Bromley B. C., Kenyon S. J., 2016, ApJ, 826, 64

Bromley B. C., Kenyon S. J., 2020, AJ, 160, 85

Brown M. E., 2001, AJ, 121, 2804

Brown M. E., 2017, AJ, 154, 65

Brown M. E., Barkume K. M., Ragozzine D., Schaller E. L., 2007, Nature, 446, 294

Brown M. E., Trujillo C., Rabinowitz D., 2004, ApJ, 617, 645

Buie M. W. et al., 2003, Earth, Moon, and Planets, 92, 113

Buitinck L. et al., 2013, in Blockeel H., Kersting K., Nijssen S., Zelezny F., eds, ECML PKDD Workshop: Languages for Data Mining and Machine Learning, Springer-Verlag Berlin Heidelberg, p. 108

Chiang E. I., Brown M. E., 1999, AJ, 118, 1411

Chiang E. I., Jordan A. B., 2002, AJ, 124, 3430

Chiang E. I., 2002, ApJ, 573, L65

Chiang E. I. et al., 2003a, AJ, 126, 430

Chiang E. I., Lovering J. R., Millis R. L., Buie M. W., Wasserman L. H., Meech K. J., 2003b, Earth, Moon, and Planets, 92, 49

Clement M. S., Sheppard S. S., 2021, AJ, in press (arXiv:2105.01065)

de la Fuente Marcos C., de la Fuente Marcos R., 2014, MNRAS, 443, L59

de la Fuente Marcos C., de la Fuente Marcos R., 2016, MNRAS, 462, 1972

de la Fuente Marcos C., de la Fuente Marcos R., 2018a, Res. Notes AAS, 2, 167

de la Fuente Marcos C., de la Fuente Marcos R., 2018b, MNRAS, 474, 838

de la Fuente Marcos C., de la Fuente Marcos R., 2021, A\&A, 646, L14

de la Fuente Marcos C., de la Fuente Marcos R., Aarseth S. J., 2015, MNRAS, 446, 1867

de la Fuente Marcos C., de la Fuente Marcos R., Aarseth S. J., 2017, Ap\&SS, 362, 198

de la Fuente Marcos C., de la Fuente Marcos R., Aarseth S. J., 2018, MNRAS, 476, L1

de León J., de la Fuente Marcos C., de la Fuente Marcos R., 2017, MNRAS, 467, L66

Dell'Oro A., Campo Bagatin A., Benavidez P. G., Alemañ R. A., 2013, A\&A, 558, A95

Dones L., 1997, in Pendleton Y. J., Tielens A. G. G. M., Savage M. L., eds, ASP Conf. Ser. Vol. 122, From Stardust to Planetesimals. Astron. Soc. Pac., San Francisco, p. 347

Fienga A., Di Ruscio A., Bernus L., Deram P., Durante D., Laskar J., Iess L., 2020, A\&A, 640, A6

Freedman D., Diaconis P., 1981, Z. Wahrscheinlichkeitstheorie verw. Gebiete, 57,453 
Gallardo T., 2006, Icarus, 184, 29

Gallardo T., 2020, Celest. Mech. Dyn. Astron., 132, 9

Ginsburg A. et al., 2019, AJ, 157, 98

Giorgini J., 2011, in Capitaine N., ed., Proceedings of the Journées 2010 "Systèmes de référence spatio-temporels" (JSR2010): New challenges for reference systems and numerical standards in astronomy, Observatoire de Paris, Paris, p. 87

Giorgini J. D., 2015, IAU General Assembly, Meeting \#29, 22, 2256293

Gladman B., Kavelaars J. J., Nicholson P. D., Loredo T. J., Burns J. A., 1998, AJ, 116, 2042

Gladman B. et al., 2001, MPEC Circ., MPEC 2001-F42

Gladman B., Holman M., Grav T., Kavelaars J., Nicholson P., Aksnes K., Petit J.-M., 2002, Icarus, 157, 269

Harris C. R. et al., 2020, Nature, 585, 357

Hills J. G., 1981, AJ, 86, 1730

Holman M. J., Payne M. J., Blankley P., Janssen R., Kuindersma S., 2018, AJ, 156, 135

Hunter J. D., 2007, CSE, 9, 90

Ito T., Ohtsuka K., 2019, Monogr. Environ. Earth Planets, 7, 1

Jewitt D., Luu J., Trujillo C., 1998, AJ, 115, 2125

Kaib N. A., Sheppard S. S., 2016, AJ, 152, 133

Kaib N. A. et al., 2019, AJ, 158, 43

Kenyon S. J., Bromley B. C., 2004, Nature, 432, 598

Kenyon S. J., Bromley B. C., 2015, ApJ, 806, 42

Kenyon S. J., Bromley B. C., 2016, ApJ, 825, 33

Kenyon S. J., Bromley B. C., 2020, PSJ, 1, 40

Khain T. et al., 2020, AJ, 159, 133

Kozai Y., 1962, AJ, 67, 591

Lan L., Malhotra R., 2019, Celest. Mech. Dyn. Astron., 131, 39

Larsen J. A. et al., 2007, AJ, 133, 1247

Levison H. F., Dones L., Duncan M. J., 2001, AJ, 121, 2253

Lidov M. L., 1962, Planet. Space Sci., 9, 719

Lloyd S. P., 1957, IEEE Transactions on Information Theory, 28, 129

Lykawka P. S., Mukai T., 2007, Icarus, 192, 238

MacQueen J. B., 1967, in Le Cam L. M., Neyman, J., eds, Proceedings of 5th Berkeley Symposium on Mathematical Statistics and Probability, Volume 1: Statistics, University of California Press, p. 281

Marcus R. A., Ragozzine D., Murray-Clay R. A., Holman M. J., 2011, ApJ, 733, 40

Millis R. L., Buie M. W., Wasserman L. H., Elliot J. L., Kern S. D., Wagner R. M., 2000, AAS/Div. Planet. Sci. Meeting Abstr., 32, 20.01

Moore N. W. H., Li G., Adams F. C., 2020, ApJ, 901, 92

Morbidelli A., Levison H. F., 2004, AJ, 128, 2564

Murray C. D., Dermott S. F., 1999, Solar System Dynamics, Cambridge Univ. Press, Cambridge, p. 71

Naess S. et al., 2021, ApJ, submitted (arXiv:2104.10264)

Napier K. J. et al., 2021, PSJ, 2, 59

Nesvorný D., Vokrouhlický D., Roig F., 2016, ApJ, 827, L35

Park R. S., Folkner W. M., Williams J. G., Boggs D. H., 2021, AJ, 161, 105

Pedregosa F. et al., 2011, Journal of Machine Learning Research, 12, 2825

Perdelwitz V., Völschow M., Müller H. M., 2018, A\&A, 615, A159

Pfalzner S., Bhandare A., Vincke K., Lacerda P., 2018, ApJ, 863, 45

Rice M., Laughlin G., 2020, European Planetary Science Congress 2020, 14, EPSC2020-420

Rickman H., Froeschlé C., Froeschlé C., Valsecchi G. B., 2004, A\&A, 428, 673

Saillenfest M., 2020, Celest. Mech. Dyn. Astron., 132, 12

Saillenfest M., Fouchard M., Tommei G., Valsecchi G. B., 2017a, Celest. Mech. Dyn. Astron., 127, 477

Saillenfest M., Fouchard M., Tommei G., Valsecchi G. B., 2017b, Celest. Mech. Dyn. Astron., 129, 329

Saillenfest M., Fouchard M., Ito T., Higuchi A., 2019, A\&A, 629, A95

Shankman C. et al., 2017, AJ, 154, 50

Sheppard S. S., Trujillo C. A., Tholen D. J., Kaib N., 2019, AJ, 157, 139

Sheppard S., Trujillo C., Tholen D., 2019, EPSC-DPS Joint Meeting 2019, 13, EPSC-DPS2019-1528

Smullen R. A., Volk K., 2020, MNRAS, 497, 1391

Steinhaus H., 1957, Bull. Acad. Polon. Sci., 4, 801
Stern S. A., 2005, AJ, 129, 526

Trujillo C. A., Sheppard S. S., 2014, Nature, 507, 471

van der Walt S., Colbert S. C., Varoquaux G., 2011, CSE, 13, 22

Virtanen P. et al., 2020, Nature Methods, 17, 261

Volk K., Malhotra R., 2017a, AJ, 154, 62

Volk K., Malhotra R., 2017b, AJ, 154, 212

Volk K. et al., 2018, AJ, 155, 260

Volk K., Malhotra R., Graham S., 2021, BAAS, 53, 5, 305.01

von Neumann J., 1951, in Householder A. S., Forsythe G. E., Germond

H. H., eds, National Bureau of Standards Applied Mathematics Series,

12 US Government Printing Office, Washington, DC, p. 36

von Zeipel H., 1910, AN, 183, 345

Wall J. V., Jenkins C. R., 2012, Practical Statistics for Astronomers. Cambridge Univ. Press, Cambridge

\section{APPENDIX A: MUTUAL NODAL DISTANCES AND UNCERTAINTY ESTIMATES}

The mutual nodal distance between two Keplerian trajectories with a common focus can be written as (see eqs. 16 and 17 in Saillenfest et al. 2017b):

$\Delta_{ \pm}=\frac{a_{2}\left(1-e_{2}^{2}\right)}{1 \pm e_{2} \cos \varpi_{2}}-\frac{a_{1}\left(1-e_{1}^{2}\right)}{1 \pm e_{1} \cos \varpi_{1}}$,

where for prograde orbits the ' + ' sign refers to the ascending node and the '-' sign to the descending one, and

$\cos \varpi_{1}=\frac{-\cos \omega_{1}\left(\sin i_{1} \cos i_{2}-\cos i_{1} \sin i_{2} \cos \Delta \Omega\right)+\sin \omega_{1} \sin i_{2} \sin \Delta \Omega}{\sqrt{1-\left(\cos i_{2} \cos i_{1}+\sin i_{2} \sin i_{1} \cos \Delta \Omega\right)^{2}}}$

and

$\cos \varpi_{2}=\frac{\cos \omega_{2}\left(\sin i_{2} \cos i_{1}-\cos i_{2} \sin i_{1} \cos \Delta \Omega\right)+\sin \omega_{2} \sin i_{1} \sin \Delta \Omega}{\sqrt{1-\left(\cos i_{2} \cos i_{1}+\sin i_{2} \sin i_{1} \cos \Delta \Omega\right)^{2}}}$,

with $\Delta \Omega=\Omega_{2}-\Omega_{1}$, and $a_{j}, e_{j}, i_{j}, \Omega_{j}$ and $\omega_{j}(j=1,2)$, are the orbital elements of the orbits involved. A small mutual nodal distance implies that in absence of protective mechanisms the objects may experience close flybys and, if the distance is small enough, even a collision. In order to obtain the actual distributions of $\Delta_{ \pm}$, we generated sets of orbital elements for the virtual ETNOs using data from Table 1. For example, the value of the semimajor axis of a virtual ETNO was computed using the expression $a_{\mathrm{v}}=a_{\mathrm{b}}+\sigma_{a} r_{\mathrm{i}}$, where $a_{\mathrm{b}}$ is the barycentric semimajor axis from Table $1, \sigma_{a}$ is the standard deviation from Table 1, and $r_{\mathrm{i}}$ is a (pseudo) random number with normal distribution computed using NumPy (van der Walt et al. 2011; Harris et al. 2020). In order to calculate statistically relevant values of $\Delta_{ \pm}$, we computed median and 16th and 84th percentiles from a set of $10^{4}$ pairs of virtual ETNOs for each actual pair from Table 1. Although the distributions are in general not normal, in a normal distribution a value that is one standard deviation above the mean is equivalent to the 84th percentile and a value that is one standard deviation below the mean is equivalent to the 16th percentile (see e.g. Wall \& Jenkins 2012). By providing these values (standard deviation and the relevant percentiles), we wanted to quantify how non-normal the distributions are for each pair. In some cases the resulting distribution is virtually normal, but in those with large uncertainties, the skewness and tails could be significant.

\section{APPENDIX B: POLES, PERIHELIA AND UNCERTAINTY ESTIMATES}

The ecliptic coordinates of the pole of an orbit are $\left(l_{\mathrm{p}}, b_{\mathrm{p}}\right)=$ $\left(\Omega-90^{\circ}, 90^{\circ}-i\right)$ and those of the longitude and latitude of an object 
at perihelion, $\left(l_{q}, b_{q}\right)$, are given by the expressions: $\tan \left(l_{q}-\Omega\right)=$ $\tan \omega \cos i$ and $\sin b_{q}=\sin \omega \sin i$ (see e.g. Murray \& Dermott 1999). The angular separations between orbital poles, $\alpha_{\mathrm{p}}$, and perihelia, $\alpha_{q}$, are given by the expressions:

$\cos \alpha_{\mathrm{p}}=\cos b_{\mathrm{p} 2} \cos b_{\mathrm{p} 1} * \cos \left(l_{\mathrm{p} 2}-l_{\mathrm{p} 1}\right)+\sin b_{\mathrm{p} 2} \sin b_{\mathrm{p} 1}$

and

$\cos \alpha_{q}=\cos b_{q 2} \cos b_{q 1} * \cos \left(l_{q 2}-l_{q 1}\right)+\sin b_{q 2} \sin b_{q 1}$,

where the subscripts 1 and 2 refer to the members of the pair. As in the case of $\Delta_{+}$, we computed median and 16th and 84th percentiles of $\alpha_{\mathrm{p}}$ and $\alpha_{q}$ from a set of $10^{4}$ pairs of virtual ETNOs for each actual pair from Table 1.

\section{APPENDIX C: BROWN DISTRIBUTION OF INCLINATIONS}

Brown $(2001,2017)$ found that the distribution of inclinations for the 'hot population' of the trans-Neptunian or Kuiper belt can be described by the expression $N(i) \propto \sin i e^{\frac{-\left(i-\mu_{i}\right)^{2}}{2 \sigma_{i}^{2}}}$, with $\mu_{i}=0^{\circ}$ and $\sigma_{i}=15^{\circ}$. In order to generate inclinations following the Brown distribution as continuous random variables, we use the universal von Neumann rejection method also known as rejection sampling (von Neumann 1951) implemented as a Python function with $\sigma_{i}=$ $14.9^{\circ}$ :

def browni(): \# rejection sampling,

$$
\text { \# von Neumann (1951) }
$$

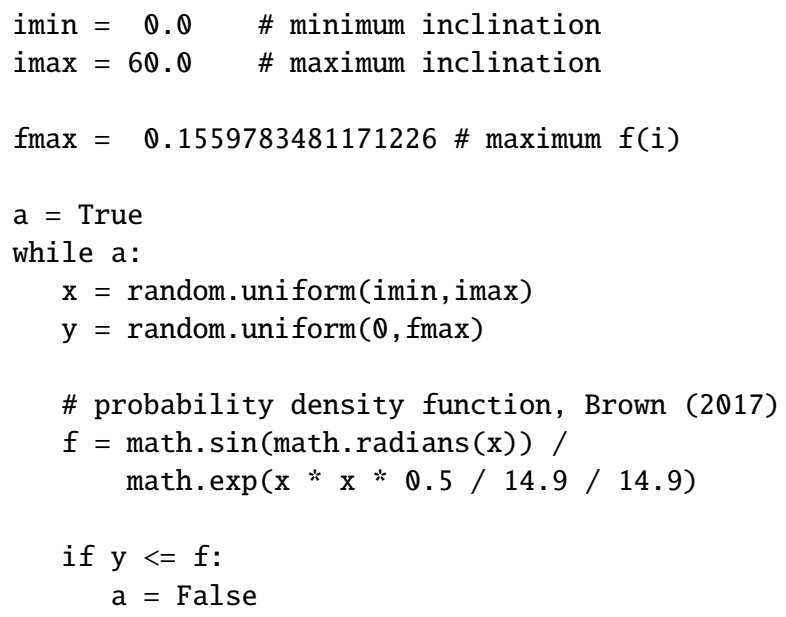

return $\mathrm{x}$

The histogram of a set of $10^{6}$ values generated with this function is shown in Fig. C1.

The other orbital elements of the virtual TNOs in Section 6.2 are generated using the expressions:

$$
\begin{aligned}
a_{\mathrm{v}} & =150+850 r_{1} \\
q_{\mathrm{v}} & =30+70 r_{2} \\
e_{\mathrm{v}} & =1.0-q_{\mathrm{v}} / a_{\mathrm{v}} \\
\Omega_{\mathrm{v}} & =360 r_{3} \\
\omega_{\mathrm{v}} & =360 r_{4},
\end{aligned}
$$

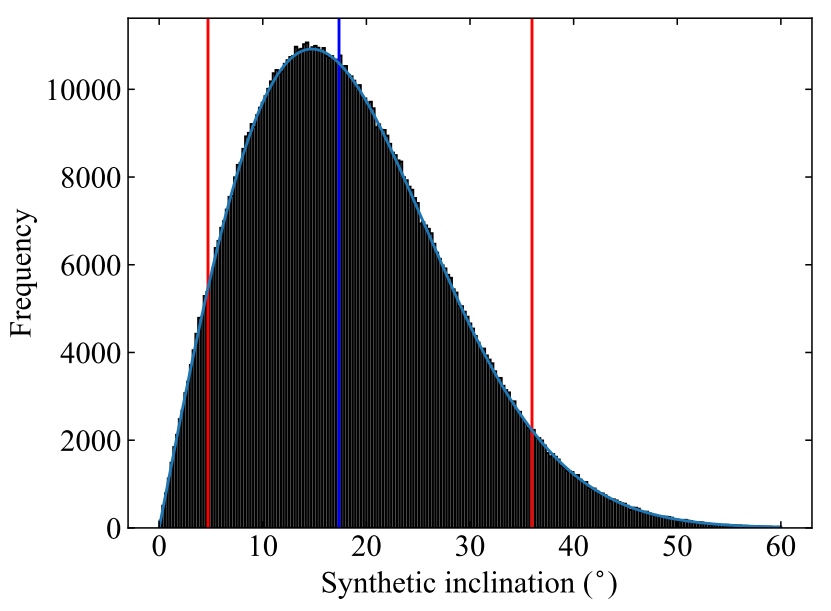

Figure C1. Distribution of inclinations consistent with the results in Brown (2001). The median is shown in blue and the 5th and 95th percentiles in red. The histogram corresponds to a sample of $10^{6}$ values. The analytical curve is shown in grey.

This paper has been typeset from a $\mathrm{T}_{\mathrm{E}} \mathrm{X} / \mathrm{L} \mathrm{A} \mathrm{E} \mathrm{X}$ file prepared by the author.

where $r_{j}$ with $j=1,4$, are random numbers in the interval $(0,1)$ with a uniform distribution. 